Article

\title{
A 4+1 Formalism for the Evolving Stueckelberg-Horwitz-Piron Metric
}

\author{
Martin Land \\ Department of Computer Science, Hadassah Academic College, Jerusalem 91010, Israel; martin@hac.ac.il \\ Received: 9 September 2020; Accepted: 15 October 2020; Published: 19 October 2020 \\ check for \\ updates
}

\begin{abstract}
We propose a field theory for the local metric in Stueckelberg-Horwitz-Piron (SHP) general relativity, a framework in which the evolution of classical four-dimensional (4D) worldlines $x^{\mu}(\tau)$ $(\mu=0,1,2,3)$ is parameterized by an external time $\tau$. Combining insights from SHP electrodynamics and the ADM formalism in general relativity, we generalize the notion of a $4 \mathrm{D}$ spacetime $\mathcal{M}$ to a formal manifold $\mathcal{M}_{5}=\mathcal{M} \times R$, representing an admixture of geometry (the diffeomorphism invariance of $\mathcal{M}$ ) and dynamics (the system evolution of $\mathcal{M}(\tau)$ with the monotonic advance of $\tau \in R)$. Strategically breaking the formal 5D symmetry of a metric $g_{\alpha \beta}(x, \tau)(\alpha, \beta=0,1,2,3,5)$ posed on $\mathcal{M}_{5}$, we obtain ten unconstrained Einstein equations for the $\tau$-evolution of the 4D metric $\gamma_{\mu \nu}(x, \tau)$ and five constraints that are to be satisfied by the initial conditions. The resulting theory differs from five-dimensional (5D) gravitation, much as SHP U(1) gauge theory differs from 5D electrodynamics.
\end{abstract}

Keywords: general relativity; Stueckelberg-Horwitz-Piron (SHP) theory; numerical relativity

\section{Introduction}

The Arnowitt Deser Misner (ADM) formalism [1] in general relativity (GR) expresses the Einstein field equations in canonical form, thus permitting a solution of particular field/matter configurations formulated as initial value problems. As a canonical Hamiltonian formulation that splits four-dimensional (4D) spacetime into three-dimensional (3D) space and a selected time direction, ADM provides insight into general features of relativity, but is not always the most convenient of the $3+1$ formulations for computation, especially numerical simulation. In this paper we borrow techniques from the 3+1 formalism in order to generalize the Stueckelberg-Horwitz-Piron (SHP) theory of classical electrodynamics [2-7] to SHP GR [8,9]. The SHP framework is a covariant canonical approach to relativistic classical and quantum mechanics, in which $4 \mathrm{D}$ spacetime events are defined with respect to coordinates $x^{\mu}(\mu=0,1,2,3)$ and an external evolution parameter $\tau$. Events trace out particle worldlines as functions $x^{\mu}(\tau)$ or $\psi(x, \tau)$ under the monotonic advance of $\tau$, producing five $\tau$-dependent gauge fields $a_{\alpha}(x, \tau)$ carrying the interaction between events. (Here and throughout the SHP literature, Greek indices $\alpha, \beta, \gamma, \ldots, \eta$ take the values $0,1,2,3,5$, while $\lambda, \mu, \nu, \ldots$ run from 0 to 3.) The result is an integrable electrodynamics, instantaneous in the external time $\tau$, but recovering Maxwell theory in a $\tau$-equilibrium limit. At numerous stages of analysis in SHP, an apparent five-dimensional (5D) symmetry arising from the five variables $x^{\mu}, \tau$ must be judiciously broken to $4+1$ representations of $\mathrm{O}(3,1)$, because the $x^{\mu}$ are coordinates while $\tau$ is an external parameter. In this paper we apply the lessons of SHP electrodynamics to a $4+1$ theory of a local metric $g_{\alpha \beta}(x, \tau)$. As we shall see, this approach differs from a $3+2$ or $(3+1)+1$ formalism, in that we do not split $4 \mathrm{D}$ spacetime into space and time, maintaining the manifest spacetime covariance of the underlying physical picture in each step. Rather, we construct a purely formal $4+1 \longrightarrow 5 \mathrm{D}$ manifold as a guide to formulating field equations that under the $5 \mathrm{D} \longrightarrow 4+1$ foliation describe a spacetime metric $\gamma_{\mu \nu}(x, \tau)$ evolving with $\tau$ and preserving the required spacetime symmetries. 


\subsection{Motivation: The Problem of Time}

In summarizing Einstein gravity as "Spacetime tells matter how to move; matter tells spacetime how to curve," Wheeler [10] touched on certain general issues in relativity known collectively as the problem of time In nonrelativistic mechanics, space is viewed as the "arena" of physical motion, a manifold with given background metric in some coordinate system, while time is an external parameter introduced to mark the coordinate evolution that characterizes the motion of objects in space. In contrast, time in general relativity retains its traditional Newtonian role as evolution parameter, but also serves as a coordinate, and thus, through the metric, plays a structural role in the spacetime "arena" itself. This dual role is complicated by the principal features of general relativity: the diffeomorphism invariance that eliminates any a priori distinction between space and time coordinates, and the background independence that regards gravitation as equivalent to motion in the spacetime determined by the local metric.

Because the metric is itself determined by the time parameterized motion of matter, practical approaches to problems in gravitation generally pose the Einstein field equations and the equations of motion for matter as an initial value problem. Beginning with a consistent spacetime geometry at some time, one may solve for the evolution of spacetime and the motions of matter over time. Known as a $3+1$ formalism, this approach singles out a time direction, as in standard Hamiltonian formulations of field theory, and so the equations are not manifestly covariant, although general covariance is preserved at each step $[1,11,12]$. On the one hand, a configuration of matter and spacetime that satisfies the equations of GR represents a $4 \mathrm{D}$ block universe, given once and describing all space, past, present, and future. Additionally, on the other hand, we may find such solutions by integrating forward in time from consistent initial conditions at some time. In Wheeler's words [13], "A decade and more of work by Dirac, Bergmann, Schild, Pirani, Anderson, Higgs, Arnowitt, Deser, Misner, DeWitt, and others has taught us through many a hard knock that Einstein's geometrodynamics deals with the dynamics of geometry: of 3-geometry, not 4-geometry."

Unsurprisingly, the foliation of spacetime into three-geometries of simultaneous points in space further complicates the interpretation of time. Because time is only felt in the evolution from one 3D submanifold to another, the Hamiltonian is constrained to vanish when restricted to any given equal-time three-geometry [12]. Moreover, there is no preferred criterion for choosing a functional of canonical variables that might be used as an intrinsic time parameter. While one may consider a physical clock that measures the proper time in some reference frame, the proper time depends on a spacetime trajectory that is only known after the equations of motion have been solved. While such a system may be well-posed in classical GR [11], this is less obvious if the metric is subject to quantum fluctuations.

\subsection{Stueckelberg-Horwitz-Piron (SHP) Theory}

Stueckelberg-Horwitz-Piron (SHP) theory is a covariant approach to relativistic classical and quantum mechanics developed to address the problem of time as it arises in electrodynamics. In 1937 Fock proposed using proper time as the evolution parameter for a Newton-like force law, succinctly expressing a manifestly covariant formulation of electrodynamics [14]. But, four years later, Stueckelberg proposed [2,3] to interpret antiparticles as particles moving backward in time, and showed that neither the coordinate time $x^{0}=c t$ nor the proper time of the motion could serve as evolution parameter for particle/antiparticle pair processes. Because $d s^{2}=\eta_{\mu v} d x^{\mu} d x^{v}$ cannot remain constant during such processes, he introduced an external time $\tau$ and argued that $d s^{2}=\eta_{\mu v} \dot{x}^{\mu} \dot{x}^{\nu} d \tau^{2}$ can be a $\tau$-dependent dynamical quantity, even in flat space. In 1973, Horwitz and Piron [4] were similarly led to use an external time in formulating a manifestly covariant relativistic mechanics with interactions, in order to overcome a priori constraints on the $4 \mathrm{D}$ phase space that conflict with canonical structure. Thus, writing the eight-dimensional (8D) unconstrained phase space

$$
x^{\mu}(\tau), \dot{x}^{\mu}(\tau) \quad \quad \dot{x}^{\mu}=\frac{d x^{\mu}}{d \tau} \quad \lambda, \mu, v, \ldots=0,1,2,3
$$


the $\mathrm{O}(3,1)$-symmetric action for a particle in Maxwell theory

$$
S_{\text {Maxwell }}=\int d \tau\left[\frac{1}{2} M \dot{x}^{\mu} \dot{x}_{\mu}+\frac{e}{c} \dot{x}^{\mu} A_{\mu}\left(x^{\lambda}\right)\right] \quad \mu, \lambda=0,1,2,3
$$

leads to the Lorentz force in the covariant form found by Fock. However, because the potential $A_{\mu}$ is produced by a Maxwell current

$$
J^{\mu}(x)=\int d \tau \dot{X}^{\mu}(\tau) \delta^{4}(x-X(\tau))
$$

depending on the trajectory $X^{\mu}(\tau)$ that is only given after the equations of motion have been solved, the system may not be well-posed. To overcome this conflict, Horwitz, Saad, and Arshansky [5] extended the action (2) by adding $\tau$-dependence to the vector potential, along with a new scalar potential, to obtain the action

$$
\begin{aligned}
S_{\text {Maxwell }} \longrightarrow S_{\text {SHP }} & =\int d \tau \frac{1}{2} M \dot{x}^{\mu} \dot{x}_{\mu}+\frac{e}{c} \dot{x}^{\mu} a_{\mu}\left(x^{\lambda}, \tau\right)+\frac{e}{c} c_{5} a_{5}\left(x^{\lambda}, \tau\right) \\
& =\int d \tau \frac{1}{2} M \dot{x}^{\mu} \dot{x}_{\mu}+\frac{e}{c} \dot{x}^{\beta} a_{\beta}\left(x^{\lambda}, \tau\right)
\end{aligned}
$$

where $\alpha, \beta, \gamma=0,1,2,3,5$, and in analogy to $x^{0}=c t$, we write $x^{5}=c_{5} \tau$. Compatibility of SHP electrodynamics with Maxwell theory requires $c_{5} \ll c$ and we will neglect $\left(c_{5} / c\right)^{2}$ where appropriate. If we take the potential to be pure gauge, as $a_{\alpha}=\partial_{\alpha} \Lambda(x, \tau)$, then the interaction term is just the total $\tau$-derivative of $\Lambda$, showing that this theory is the most general $\mathrm{U}(1)$ gauge theory on the unconstrained phase space (see also [15]). Variation with respect to $x^{\mu}$ leads to the Lorentz force [16] in the form

$$
\begin{aligned}
M \ddot{x}_{\mu} & =\frac{e}{c}\left(\dot{x}^{v} f_{\mu \nu}+c_{5} f_{\mu 5}\right)=\frac{e}{c} \dot{x}^{\beta} f_{\mu \beta} \\
\frac{d}{d \tau}\left(-\frac{1}{2} M \dot{x}^{\mu} \dot{x}_{\mu}\right) & =c_{5} \frac{e}{c} \dot{x}^{\beta} f_{5 \beta}
\end{aligned}
$$

where the field

$$
f_{\alpha \beta}=\partial_{\alpha} a_{\beta}-\partial_{\beta} a_{\alpha}
$$

is made a dynamical quantity by addition of a kinetic term of the type

$$
S_{\text {field }}=\int d \tau d^{4} x f^{\alpha \beta}(x, \tau) f_{\alpha \beta}(x, \tau)
$$

to the total action. Because the apparent 5D symmetry of the interaction term $\dot{x}^{\beta} a_{\beta}(x, \tau)$ in the action (5) is broken to 4+1 in (4), SHP electrodynamics differs in significant ways from 5D Maxwell theory. We notice that (7) permits the exchange of mass between particles and fields, and indicates the condition for non-conservation of proper time. It has been shown [16] that the total mass, energy, and momentum of particles and fields are conserved.

These equations of motion, along with the $\tau$-dependent field equations, have been used to calculate [17] the Bethe-Heitler mechanism for electron-positron production in classical electrodynamics. A positron (an electron with $\dot{x}^{0}=c \dot{t}<0$ ) propagates backward in coordinate time until entering the bremsstrahlung field produced by another electron scattering off a heavy nucleus. This field leads to $\ddot{t}>0$, so the particle gains energy $E=M c^{2} \dot{t}<0$ continuously (and thus $\dot{x}^{\mu} \dot{x}_{\mu}$ changes sign twice) until emerging as an electron propagating forward in coordinate time with $E=M c^{2} \dot{t}>0$. At coordinate times prior to the particle's turn-around (when $E=M c^{2} \dot{t}=0$ ) no particles will be observed, but two particles will be observed for subsequent coordinate times, implementing Stueckelberg's picture of pair creation. 
A physical event $x^{\mu}(\tau)$ in SHP is an irreversible occurrence at time $\tau$ with spacetime coordinates $x^{\mu}$. The formalism thereby implements the two aspects of time as distinct physical quantities: the coordinate time $x^{0}=c t$ describing the locations of events, and the external Stueckelberg time $\tau$ describing the chronological order of event occurrence. This eliminates grandfather paradoxes because for $\tau_{2}>\tau_{1}$ an event $x^{\mu}\left(\tau_{2}\right)$ at some spacetime point $x^{\mu}$ occurs after the event $x^{\mu}\left(\tau_{1}\right)$ and cannot affect it. Similarly, the $4 \mathrm{D}$ block universe $\mathcal{M}(\tau)$ occurs at $\tau$, representing the $4 \mathrm{D}$ manifold of general relativity, comprising all of space and coordinate time $x^{0}$. A Hamiltonian $K$ generates evolution of $\mathcal{M}(\tau)$ occurring at $\tau$ to an infinitesimally close 4 D block universe $\mathcal{M}(\tau+d \tau)$ occurring at $\tau+d \tau$. The configuration of spacetime, including the past and future of $x^{0}=c t$, may thus change infinitesimally from chronological moment to moment in $\tau$. Thus, it is not unreasonable to expect that $\mathcal{M}(\tau)$ will be endowed with a $\tau$-dependent metric $\gamma_{\mu \nu}(x, \tau)$ whose dynamics we explore in this paper. On the contrary, a $4 \mathrm{D}$ metric given for all $\tau$ would have the character of an absolute background field in this formalism, in violation of the goals of general relativity.

For the kinetic term (9) we formally raise the five-index of $f_{\alpha \beta}$ although we understand the Lagrangian density as

$$
f^{\alpha \beta}(x, \tau) f_{\alpha \beta}(x, \tau)=f^{\mu v}(x, \tau) f_{\mu \nu}(x, \tau)+2 \sigma f_{5}^{\mu}(x, \tau) f_{\mu 5}(x, \tau)
$$

with $\sigma= \pm 1$ simply the choice of sign for the vector-vector term. That is, we bear in mind that in this notation the $\beta=5$ index is a formal convenience, indicating $\mathrm{O}(3,1)$ scalar quantities, not an element of a 5D tensor, and not a timelike coordinate. In particular, $\dot{x}^{5}=c_{5}$ is constrained to be a constant scalar, identical in all reference frames, and $x^{5}=c_{5} \tau$ must not be treated as a dynamical variable. Nevertheless, the contraction on indices $\alpha, \beta$ suggests a formal $5 \mathrm{D}$ symmetry, possibly $\mathrm{O}(4,1)$ or $\mathrm{O}(3,2)$ that breaks to $\mathrm{O}(3,1)$ in the presence of matter, and for convenience we write

$$
\eta_{\alpha \beta}=\operatorname{diag}(-1,1,1,1, \sigma)
$$

in the form of a $5 \mathrm{D}$ flat space metric. Although the higher symmetry is non-physical for matter, it appears in wave equations, much as the wave equations for nonrelativistic acoustics appear to possess a Lorentz symmetry not associated with the physics. In developing an SHP approach to general relativity, we will similarly exploit this notation as a guide to the appropriate extension of GR while respecting the non-dynamical character of $x^{5}$.

Classical and quantum SHP particle mechanics in a spacetime with a $\tau$-independent local metric $\gamma_{\mu v}(x)$ has been studied extensively by Horwitz $[8,9]$ and will not be discussed at length here. Our goal in this paper is to find a consistent prescription for extending general relativity to accommodate a metric $g_{\alpha \beta}(x, \tau)$ (where $\alpha, \beta=0,1,2,3,5$ ) satisfying $\tau$-dependent Einstein equations on a formal 5D manifold whose meaning is explored through particle mechanics and field equations. As in standard approaches to GR, the study of embedded hypersurfaces is central to this program. But, while the $3+1$ formalism begins with a $4 \mathrm{D}$ block universe $\mathcal{M}$ and defines a foliation into embedded spacelike hypersurfaces of equal coordinate time $t$, the $4+1$ formalism begins with a parameterized family of $4 \mathrm{D}$ spacetimes $\mathcal{M}(\tau)$ embedded as hypersurfaces into a $5 \mathrm{D}$ pseudo-spacetime. Because the evolution of $\mathcal{M}(\tau)$ is determined by an $\mathrm{O}(3,1)$ scalar Hamiltonian $K$, with $\tau$ as an external parameter (Poincaré invariant by definition), there is no conflict with the diffeomorphism invariance of general relativity. This approach will guide us toward the formal structures of a $5 \mathrm{D}$ manifold $\mathcal{M}_{5}$ with coordinates $(x, \tau)$ on which we may perform a $4+1$ foliation by choosing $\tau$ as the unambiguously preferred time direction (See $[18,19]$ for discussion of general 5D spacetime with preferred foliation.). We refer to $\mathcal{M}_{5}$ as a pseudo-spacetime to emphasize that despite the formal manifold structure, in specifying the physics we treat $\tau$ as a parameter and not a coordinate. Moreover, $\mathcal{M}_{5}$ represents an admixture of symmetries: 4D spacetime geometry within each $\mathcal{M}(\tau)$, and canonical dynamics between any pair $\mathcal{M}\left(\tau_{1}\right), \mathcal{M}\left(\tau_{2}\right)$. We expect no general diffeomorphism invariance for $\mathcal{M}_{5}$. 


\subsection{Organization of This Paper}

The remainder of this paper is organized, as follows: in Section 2, we formulate the particle mechanics for an event in 5D pseudo-spacetime, derive the 5D mass-energy-momentum tensor for non-thermodynamic dust, and pose the Einstein field equations generalized to 5D. We obtain a general solution for the associated weak field equations, and consider a source event of slightly varying mass (time acceleration in a co-moving frame). This leads to a small nonrelativistic modification to Newtonian gravity in which the mass variation of the source is transferred through the metric to induce varying mass motion in a test event. In Section 3, we formalize the foliation of the 5D pseudo-spacetime into the $4+1$ hypersurface geometry, and by projecting onto tangent and normal components, express 5D Einstein equations as a set of coupled partial differential equations in the intrinsic and extrinsic curvature of the hypersurface. In Section 4, we complete the 4+1 ADM formalism by transforming the differential equations to covariant canonical Hamiltonian form. Finally, in Section 5 we apply the $4+1$ formalism to two possible generalizations of Schwarzschild geometry. In the first, we include a non-trivial fifth component in the diagonal metric, which is seen to be constrained to satisfy a $4 \mathrm{D}$ wave equation. A test event moving in the resulting field evolves with mass that depends on its distance from the source. In the second, we allow for the mass parameter in the standard Schwarzschild metric to be $\tau$-dependent and find the conditions of the mass-energy-momentum tensor that lead to such a solution. The presented examples were chosen because they can be solved in closed form. Realistic applications of this formalism will necessarily require numerical solutions beyond the scope of this paper.

\section{Particle Mechanics}

\subsection{Particle Lagrangian in Standard GR}

Regarding the spacetime manifold $\mathcal{M}$ as a $4 \mathrm{D}$ block universe, general relativity begins with consideration of the squared interval

$$
\delta x^{2}=\gamma_{\mu \nu} \delta x^{\mu} \delta x^{\nu}=\left(x_{2}-x_{1}\right)^{2}
$$

between two neighboring points of $\mathcal{M}$. The invariance of this interval, viewed as an instantaneous displacement in the block universe, is a geometrical statement referring to the freedom that is permitted in assigning a coordinate map to the manifold. To extract dynamics from geometry, one considers the spacetime trajectory of a material event (some appropriate abstraction of point mass, which in GR would necessarily be a black hole), described as a mapping of an arbitrary parameter $\zeta$ to a continuous sequence of events $x^{\mu}(\zeta)$ in $\mathcal{M}$. Because the interval between any two points on a trajectory must be timelike, the proper time $s$ may be taken as parameter, and "motion" along the trajectory is observed through advances in the time coordinate $x^{0}(s)$ for advancing values of $s$. The invariant interval (12) can be written

$$
\delta x^{2}=\gamma_{\mu \nu} \delta x^{\mu} \delta x^{v}=\gamma_{\mu \nu} \frac{d x^{\mu}}{d s} \frac{d x^{v}}{d s} \delta s^{2}=\gamma_{\mu \nu} \dot{x}^{\mu} \dot{x}^{v} \delta s^{2}
$$

suggesting [20] a dynamical description of the trajectory by the action

$$
S=\int d x=\int d s \sqrt{-\gamma_{\mu \nu} \dot{x}^{\mu} \dot{x}^{v}}
$$

and leading to geodesic equations of motion as an expression of the equivalence principle. The geodesic equations can also be derived from the action

$$
S=\int d s \frac{1}{2} \gamma_{\mu v} \dot{x}^{\mu} \dot{x}^{v}
$$

which removes the constraint $\dot{x}^{2}=-c^{2}$ associated with (14). 


\subsection{Particle Lagrangian in SHP GR}

To extend the SHP classical mechanics of a free particle to a manifold with a $\tau$-dependent local metric, we begin by considering the interval

$$
d x^{\mu}=x_{1}^{\mu}\left(\tau_{1}\right)-x_{2}^{\mu}\left(\tau_{2}\right)
$$

between an event $x_{1}^{\mu} \in \mathcal{M}\left(\tau_{1}\right)$ and an event $x_{2}^{\mu} \in \mathcal{M}\left(\tau_{2}\right)$. Writing these events as

$$
X_{1}=\left(x_{1}, c_{5} \tau_{1}\right) \quad X_{2}=\left(x_{2}, c_{5} \tau_{2}\right)
$$

we introduce a notion of 5D distance by combining the geometrical distance $\delta x$ between any two arbitrary points in $\mathcal{M}(\tau)$, with the dynamical distance between events generated by a Hamiltonian that evolves $\mathcal{M}(\tau) \longrightarrow \mathcal{M}(\tau+\delta \tau)$. The geometrical distance is characterized by the squared relativistic interval (12) and taking $\tau_{2}=\tau_{1}+\delta \tau$, so that

$$
x_{2}\left(\tau_{1}+\delta \tau\right)-x_{1}\left(\tau_{1}\right) \simeq x_{2}\left(\tau_{1}\right)+\frac{d x(\tau)}{d \tau} \delta \tau-x_{1}\left(\tau_{1}\right)=\delta x+\frac{d x(\tau)}{d \tau} \delta \tau
$$

and we write the difference in the form

$$
X_{2}-X_{1}=\left(\delta x+\frac{d x(\tau)}{d \tau} \delta \tau, c_{5} \delta \tau\right)
$$

which motivates the notion of a 5D invariant interval through

$$
d X^{2}=\gamma_{\mu \nu}\left(\delta x^{\mu}+\frac{d x^{\mu}(\tau)}{d \tau} \delta \tau\right)\left(\delta x^{\nu}+\frac{d x^{\nu}(\tau)}{d \tau} \delta \tau\right)+\sigma c_{5}^{2} \delta \tau^{2}=g_{\alpha \beta}(x, \tau) \delta x^{\alpha} \delta x^{\beta}
$$

referred to $x_{1}$ coordinates at $\tau=\tau_{1}$. Because the manifold $\mathcal{M}(\tau)$ evolves, the spacetime metric $\gamma_{\mu v}$ must depend on $x$ and $\tau$ in some manner to be determined.

As in $4 \mathrm{D}$ general relativity, the squared interval (20) suggests the Lagrangian

$$
L=\frac{1}{2} M g_{\alpha \beta}\left(x^{\mu}, x^{5}\right) \dot{x}^{\alpha} \dot{x}^{\beta} \quad \lambda, \mu, v=0,1,2,3 \quad \alpha, \beta, \gamma=0,1,2,3,5
$$

from which we may find equations of motion in the space determined by the local metric $g_{\alpha \beta}$.

\subsection{Equations of Motion}

Before examining particle dynamics in SHP GR, we consider a straightforward extension of GR to unbroken $5 \mathrm{D}$, with coordinates $x^{\alpha}$, for $\alpha=0,1,2,3,5$ and external evolution parameter $\tau$. Naively applying the Euler-Lagrange equations to the action (21), posing no fixed relationship between $x^{5}$ and $\tau$, we find

$$
0=\frac{d}{d \tau} \frac{\partial L}{\partial \dot{x} \gamma}-\frac{\partial L}{\partial x^{\gamma}}=\frac{d}{d \tau}\left(g_{\alpha \gamma} \dot{x}^{\alpha}\right)-\frac{1}{2} \frac{\partial}{\partial x^{\gamma}} g_{\alpha \beta} \dot{x}^{\alpha} \dot{x}^{\beta}
$$

leading to the five geodesic equations

$$
0=\frac{D \dot{x}^{\gamma}}{D \tau}=\ddot{x}^{\gamma}+\Gamma_{\alpha \beta}^{\gamma} \dot{x}^{\alpha} \dot{x}^{\beta}
$$

where $D / D \tau$ is the absolute derivative (in the notation of Weinberg [21]) and

$$
\Gamma_{\alpha \beta}^{\gamma}=g^{\gamma \delta} \Gamma_{\delta \alpha \beta}=\frac{1}{2} g^{\gamma \delta}\left(\partial_{\alpha} g_{\delta \beta}+\partial_{\beta} g_{\delta \alpha}-\partial_{\delta} g_{\beta \alpha}\right)
$$

is the standard Christoffel symbol in 5D. Writing the canonical momentum 


$$
p_{\alpha}=\frac{\partial L}{\partial \dot{x}^{\alpha}}=M g_{\alpha \beta} \dot{x}^{\beta} \quad \longrightarrow \quad \dot{x}^{\alpha}=\frac{1}{M^{\alpha}} g^{\alpha \beta} p_{\beta}
$$

the Hamiltonian

$$
K=\dot{x}^{\alpha} p_{\alpha}-L=\frac{1}{2 M} g^{\alpha \beta} p_{\alpha} p_{\beta}=L
$$

is conserved, as seen directly through

$$
\frac{d}{d \tau}\left(\frac{1}{2} M g_{\alpha \beta} \dot{x}^{\alpha} \dot{x}^{\beta}\right)=M g_{\alpha \beta} \dot{x}^{\alpha} \frac{D \dot{x}^{\beta}}{D \tau}=0
$$

where we used metric compatibility

$$
\frac{D g_{\alpha \beta}}{D \tau}=0
$$

Time independence of the Hamiltonian may also be found from the canonical equations of motion

$$
\dot{x}^{\alpha}=\frac{d x^{\alpha}}{d \tau}=\frac{\partial K}{\partial p_{\alpha}} \quad \dot{p}_{\alpha}=\frac{d p_{\alpha}}{d \tau}=-\frac{\partial K}{\partial x^{\alpha}}
$$

and the Poisson bracket

$$
\{F, G\}=\frac{\partial F}{\partial x^{\alpha}} \frac{\partial G}{\partial p_{\alpha}}-\frac{\partial F}{\partial p_{\alpha}} \frac{\partial G}{\partial x^{\alpha}}
$$

so that

$$
\frac{d}{d \tau}\left(\frac{1}{2 M} g^{\alpha \beta} p_{\alpha} p_{\beta}\right)=\frac{d K}{d \tau}=\{K, K\}+\frac{\partial K}{\partial \tau}=\frac{1}{2 M} p_{\alpha} p_{\beta} \frac{\partial g^{\alpha \beta}}{\partial \tau}=0
$$

because the metric is not explicitly dependent on $\tau$, which in this case bears no specific relationship with $x^{5}$. As seen in SHP electrodynamics, the equation

$$
0=\frac{D \dot{x}^{5}}{D \tau}=\ddot{x}^{5}+\Gamma_{\alpha \beta}^{5} \dot{x}^{\alpha} \dot{x}^{\beta}
$$

cannot generally be made consistent with the SHP condition $x^{5}=c_{5} \tau \Rightarrow \ddot{x}^{5}=0$. Rather, the SHP formalism defines $x^{5}$ to be a scalar, in which case the absolute derivative reduces to the total derivative, so that

$$
\frac{D \dot{x}^{5}}{D \tau}=\frac{d \dot{x}^{5}}{d \tau}=0
$$

will replace (32).

To obtain the correct equations of motion for SHP, we must break the 5D symmetry of (21) to 4+1 prior to applying the Euler-Lagrange equations and not treat $x^{5}$ as a dynamical quantity. Expanding

$$
L=\frac{1}{2} M g_{\alpha \beta}(x, \tau) \dot{x}^{\alpha} \dot{x}^{\beta}=\frac{1}{2} M g_{\mu \nu} \dot{x}^{\mu} \dot{x}^{v}+M c_{5} g_{\mu 5} \dot{x}^{\mu}+\frac{1}{2} M c_{5}^{2} g_{55}
$$

the equations of motion have four components

$$
\ddot{x}^{\mu}+\Gamma_{\lambda \sigma}^{\mu} \dot{x}^{\lambda} \dot{x}^{\sigma}+2 c_{5} \Gamma_{5 \sigma}^{\mu} \dot{x}^{\sigma}+c_{5}^{2} \Gamma_{55}^{\mu}=0
$$

and, because $x^{5}$ is not a dynamical quantity, it has no conjugate momentum. Thus, while (35) is identical to (23) for $\mu=0,1,2,3$, we understand (32) in the sense of (33). The breaking of 5D symmetry is expressed here in that $\Gamma_{\alpha \beta}^{5}$ can be calculated, but it plays no part in the equations of motion. The 4-momentum is 


$$
p_{\mu}=\frac{\partial L}{\partial \dot{x}^{\mu}}=M g_{\mu v} \dot{x}^{v}+M c_{5} g_{\mu 5} \quad \longrightarrow \quad \dot{x}_{\mu}=\frac{1}{M}\left(p_{\mu}-M c_{5} g_{\mu 5}\right)
$$

allowing us to write the Hamiltonian in the form

$$
K=p_{\mu} \dot{x}^{\mu}-L=\left(M g_{\mu \nu} \dot{x}^{\nu}+M c_{5} g_{\mu 5}\right) \dot{x}^{\mu}-L=\frac{1}{2} M g_{\mu \nu} \dot{x}^{\mu} \dot{x}^{v}-\frac{1}{2} M c_{5}^{2} g_{55}
$$

which, unlike the Hamiltonian for unbroken 5D symmetry, is not equal to the Lagrangian (The difference is precisely the term $p_{5} \dot{x}^{5}$ that would be present in the Legendre transformation if we had taken $x^{5}$ to be dynamical). Taking the total $\tau$-derivative of (37) and inserting the equations of motion (35) leads to

$$
\frac{d K}{d \tau}=-\frac{1}{2} M \dot{x}^{\mu} \dot{x}^{\nu} \frac{\partial g_{\mu v}}{\partial \tau}-\frac{1}{2} M c_{5}^{2} \frac{\partial g_{55}}{\partial \tau}
$$

showing that this Hamiltonian is not conserved for a $\tau$-dependent metric. Using (36) to eliminate $\dot{x}_{\mu}$, we put the Hamiltonian into the form

$$
K=\frac{1}{2 M} g^{\mu v} p_{\mu} p_{v}-c_{5} g_{5}^{\mu} p_{\mu}+\frac{1}{2} M c_{5}^{2}\left(g_{5}^{\mu} g_{\mu 5}-g_{55}\right)
$$

and find its non-conservation from the Poisson bracket

$$
\frac{d K}{d \tau}=\{K, K\}+\frac{\partial K}{\partial \tau}=-\frac{1}{2 M} p^{\mu} p^{v} \frac{\partial g_{\mu v}}{\partial \tau}-c_{5} p_{\mu} \frac{\partial g_{\mu 5}}{\partial \tau}+\frac{1}{2} M c_{5}^{2}\left(2 g_{5}^{\mu} \frac{\partial g_{\mu 5}}{\partial \tau}-\frac{\partial g_{55}}{\partial \tau}\right)
$$

where we used

$$
\frac{\partial g^{\mu \nu}}{\partial \tau}=-g^{\mu \rho} g^{v \sigma} \frac{\partial g_{\rho \sigma}}{\partial \tau} .
$$

When $g_{\alpha 5}=0$, the Hamiltonian (39) is seen to generalize the nonrelativistic expression $\mathbf{p}^{2} / 2 m$ for the energy of a free particle. Because $K$ is a Lorentz scalar, SHP theory associates this Hamiltonian with the dynamical mass of the particle motion. Section 2.5 provides an example of a test particle evolving with variable mass in a $\tau$-dependent local metric.

\subsection{Mass-Energy-Momentum Tensor}

When considering non-thermodynamic dust, we define $n(x, \tau)$ to be the number of events per spacetime volume, and

$$
j^{\alpha}(x, \tau)=\rho(x, \tau) \dot{x}^{\alpha}(\tau)=M n(x, \tau) \dot{x}^{\alpha}(\tau)
$$

is the five-component event current. The continuity equation in flat space is

$$
\partial_{\alpha} j^{\alpha}=\partial_{\mu} j^{\mu}+\partial_{5} j^{5}=\partial_{\mu} j^{\mu}+\frac{\partial \rho}{\partial \tau}=0
$$

and with a local metric is generalized to

$$
\nabla_{\alpha} j^{\alpha}=0
$$

where (in the notation of Wald [22]), the covariant derivative for a vector is

$$
\nabla_{\alpha} X^{\beta}=\frac{\partial X^{\beta}}{\partial x^{\alpha}}+X^{\gamma} \Gamma_{\gamma \alpha}^{\beta} .
$$

But again, since $j^{5}$ is a scalar (the number density is scalar on physical grounds) for which the covariant derivative is just the partial derivative, we must have 


$$
\nabla_{5} j^{5}=\frac{\partial \rho}{\partial \tau}
$$

so the continuity equation becomes

$$
\frac{\partial \rho}{\partial \tau}+\nabla_{\mu} j^{\mu}=0
$$

Generalizing the 4D stress-energy-momentum tensor to 5D, we write the mass-energy-momentum tensor [23] as

$$
T^{\alpha \beta}=\rho \dot{x}^{\alpha} \dot{x}^{\beta} \longrightarrow\left\{\begin{array}{l}
T^{\mu v}=\rho \dot{x}^{\mu} \dot{x}^{v} \\
T^{5 \beta}=c_{5} j^{\beta}
\end{array}\right.
$$

where, in addition to the $4 \mathrm{D}$ components $T^{\mu v}$, we have the current density $T^{5 \beta}=\dot{x}^{5} \dot{x}^{\beta} \rho=c_{5} j^{\beta}$. The conservation equation is

$$
0=\nabla_{\beta} T^{\alpha \beta}=\nabla_{\beta}\left(\rho \dot{x}^{\alpha} \dot{x}^{\beta}\right)=\dot{x}^{\alpha} \nabla_{\beta}\left(\rho \dot{x}^{\beta}\right)+\rho \dot{x}^{\beta} \nabla_{\beta} \dot{x}^{\alpha}=\dot{x}^{\alpha} \nabla_{\beta} j^{\beta}+\rho \dot{x}^{\beta} \nabla_{\beta} \dot{x}^{\alpha}
$$

which vanishes by virtue of the continuity and geodesic equations

$$
\nabla_{\alpha} j^{\alpha}=0 \quad \dot{x}^{\beta} \nabla_{\beta} \dot{x}^{\alpha}=\frac{D \dot{x}^{\alpha}}{D \tau}=0
$$

when the equations of motion (23) are evaluated in the sense of (33).

\subsection{Weak Field Approximation}

As a first step in obtaining field equations for $g_{\alpha \beta}$ we extend the Einstein equations to $5 \mathrm{D}$ as

$$
G_{\alpha \beta}=R_{\alpha \beta}-\frac{1}{2} R g_{\alpha \beta}=\frac{8 \pi G}{c^{4}} T_{\alpha \beta}
$$

where the Ricci tensor $R_{\alpha \beta}$ and scalar $R$ are obtained by contracting indices of the $5 \mathrm{D}$ curvature tensor $R_{\gamma \alpha \beta}^{\delta}$. The weak field approximation (see for example $[20,24,25]$ ) is generalized to SHP GR by introducing a perturbation $h_{\alpha \beta}$ to the flat metric, such that

$$
g_{\alpha \beta}=\eta_{\alpha \beta}+h_{\alpha \beta} \longrightarrow \partial_{\gamma} g_{\alpha \beta}=\partial_{\gamma} h_{\alpha \beta} \quad\left(h_{\alpha \beta}\right)^{2} \approx 0
$$

leading to the Ricci tensor

$$
R_{\alpha \beta} \simeq \frac{1}{2}\left(\partial_{\beta} \partial_{\gamma} h_{\alpha}^{\gamma}+\partial_{\alpha} \partial_{\gamma} h_{\beta}^{\gamma}-\partial_{\gamma} \partial_{\gamma} h_{\alpha \beta}-\partial_{\alpha} \partial_{\beta} h\right) \quad R \simeq \eta^{\alpha \beta} R_{\alpha \beta} \quad h \simeq \eta^{\alpha \beta} h_{\alpha \beta}
$$

which naturally contains only the perturbation. Defining $\bar{h}_{\alpha \beta}=h_{\alpha \beta}-\frac{1}{2} \eta_{\alpha \beta} h$, the Einstein equations become

$$
\frac{16 \pi G}{c^{4}} T_{\alpha \beta}=\partial_{\beta} \partial_{\gamma} \bar{h}_{\alpha}^{\gamma}+\partial_{\alpha} \partial_{\gamma} \bar{h}_{\beta}^{\gamma}-\partial_{\gamma} \partial_{\gamma} \bar{h}_{\alpha \beta}-\partial_{\alpha} \partial_{\beta} \bar{h}
$$

which take the form of a wave equation

$$
\frac{16 \pi G}{c^{4}} T_{\alpha \beta}=-\partial^{\gamma} \partial_{\gamma} \bar{h}_{\alpha \beta}=-\left(\partial^{\mu} \partial_{\mu}+\frac{\eta_{55}}{c_{5}^{2}} \partial_{\tau}^{2}\right) \bar{h}_{\alpha \beta}
$$

by imposing the usual gauge condition $\partial_{\lambda} \bar{h}^{\alpha \lambda}=0$. The principal part Green's function [26] for this wave equation is

$$
G(x, \tau)=-\frac{1}{2 \pi} \delta\left(x^{2}\right) \delta(\tau)-\frac{c_{5}}{2 \pi^{2}} \frac{\partial}{\partial x^{2}} \theta\left(-\eta_{55} g_{\alpha \beta} x^{\alpha} x^{\beta}\right) \frac{1}{\sqrt{-\eta_{55} g_{\alpha \beta} x^{\alpha} x^{\beta}}}
$$


in which the first term is dominant at long distance, leading to the solution

$$
\bar{h}_{\alpha \beta}(x, \tau)=\frac{4 G}{c^{4}} \int d^{3} x^{\prime} \frac{T_{\alpha \beta}\left(t-\frac{\left|\mathbf{x}-\mathbf{x}^{\prime}\right|}{c}, \mathbf{x}^{\prime}, \tau\right)}{\left|\mathbf{x}-\mathbf{x}^{\prime}\right|}
$$

relating the field $\bar{h}_{\alpha \beta}(x, \tau)$ to the source $T_{\alpha \beta}(x, \tau)$.

As a simple example, we consider a source $X=(c T(\tau), 0)$ in a co-moving frame, so that $\dot{T} \neq$ constant corresponds to a variation in energy without corresponding variation in momentum, producing a variation in mass. The non-zero components of the mass-energy-momentum tensor are

$$
T^{00}=m c^{2} \dot{T}^{2} \delta^{3}(\mathbf{x}) \rho(t-T(\tau)) \quad T^{\alpha i}=0 \quad T^{55}=\frac{c_{5}^{2}}{c^{2}} T^{00} \approx 0
$$

where we neglect $c_{5}^{2} / c^{2} \ll 1$ and have written $M(\tau)=m \rho(t-T(\tau))$ to represent a slowly varying density function (the source is sharply located in space but smeared along the $t$-axis). The perturbed metric is found to be

$$
\bar{h}^{00}(x, \tau)=\frac{4 G M}{c^{2} R} \dot{T}^{2} \quad \bar{h}^{\alpha i}(x, \tau)=0 \quad \bar{h}^{55}(x, \tau)=0
$$

so using $h_{\alpha \beta}=\bar{h}_{\alpha \beta}-\frac{1}{2} \eta_{\alpha \beta} \bar{h}$, we see that $h^{00}=\bar{h}^{00}$. Since $g^{\alpha \beta} h_{\beta \gamma} \simeq \eta^{\alpha \beta} h_{\beta \gamma}$ the non-zero Christoffel symbols are

$$
\Gamma_{00}^{\mu}=-\frac{1}{2} \eta^{\mu v} \partial_{v} h_{00} \quad \Gamma_{0 i}^{\mu}=\frac{1}{2} \eta^{\mu v} \partial_{i} h_{v 0} \quad \Gamma_{50}^{\mu}=\frac{1}{2 c_{5}} \eta^{\mu 0} \partial_{\tau} h_{00}
$$

and the equations of motion for a distant test particle split into

$$
\ddot{t}=\left(\partial_{\tau} h_{00}\right) \dot{t}+\dot{\mathbf{x}} \cdot\left(\nabla h_{00}\right) \dot{t}^{2} \quad \ddot{\mathbf{x}}=\frac{c^{2}}{2}\left(\nabla h_{00}\right) \dot{t}^{2}
$$

where the factor $\partial_{\tau} h_{00}$ distinguishes these equations from the Newtonian model. We write the space part in spherical coordinates, putting $\theta=\pi / 2$, so that the angular and radial equations become

$$
2 \dot{R} \dot{\phi}+R \ddot{\phi}=0 \longrightarrow \dot{\phi}=\frac{L}{M R^{2}} \longrightarrow \ddot{R}-\frac{L^{2}}{M^{2} R^{3}}=-\frac{G M}{R^{2}} \dot{t}^{2} \dot{T}^{2}
$$

where $L$ is a constant of integration with units of angular momentum. Introducing $\alpha(\tau)$ through

$$
\dot{T}=1+\frac{\alpha(\tau)}{2} \longrightarrow \dot{T}^{2} \simeq 1+\alpha(\tau) \longrightarrow \dot{T} \ddot{T} \simeq\left(1+\frac{\alpha(\tau)}{2}\right) \frac{\dot{\alpha}(\tau)}{2}
$$

the relationship between $t$ and $\tau$ becomes

$$
\ddot{t}=\frac{2 G \partial_{\tau} M}{c^{2} R} \dot{t}+\frac{4 G M}{c^{2} R} \dot{T} \ddot{T} \dot{t}-\frac{2 G M}{R^{2} c^{2}} \dot{R} \dot{T}^{2} \approx \frac{2 G M}{c^{2} R}\left(1+\frac{\alpha(\tau)}{2}\right) \dot{\alpha}(\tau) \dot{t}
$$

where we neglect the nonrelativistic velocity $\dot{R} / c \approx 0$ and the slow variation in the source distribution $\partial_{\tau} \rho \approx 0$. In the absence of the mass perturbation, we have $\alpha=0 \longrightarrow \dot{t}=1$, recovering a Newtonian notion of time, but this $t$ equation has the solution

$$
\dot{t}=\exp \left[\frac{2 G M}{c^{2} R}\left(\alpha+\frac{1}{4} \alpha^{2}\right)\right] \longrightarrow \dot{t}^{2} \dot{T}^{2} \simeq 1+\left(1+\frac{4 G M}{c^{2} R}\right) \alpha
$$

indicating a more complicated relationship between $t$ and $\tau$. Since $4 G M / c^{2} R \ll 1$, this leads finally to a radial equation in the form

$$
\frac{d}{d \tau}\left\{\frac{1}{2} \dot{R}^{2}+\frac{1}{2} \frac{L^{2}}{M^{2} R^{2}}-\frac{G M}{R}[1+\alpha(\tau)]\right\}=\frac{d K}{d \tau}=-\frac{G M}{R} \frac{d}{d \tau} \alpha(\tau) .
$$


We recognize $K$ on the LHS as the Hamiltonian of the test particle moving in this local metric, recovering the Newtonian expression when the perturbation $\alpha(\tau)$ vanishes. The mass fluctuation of the point source is seen to induce a fluctuation in the mass of the distant test particle, acting through the field $g_{\alpha \beta}(x, \tau)$ in order to produce a small modification of Newtonian gravity.

\section{Field Equations}

In a $3+1$ formalism such as ADM, a spacetime trajectory is defined with respect to a foliation of $\mathcal{M}$. For any point $x^{\mu} \in \mathcal{M}$, we define a time function $t(x)$ on $\mathcal{M}$ whose level sets

$$
\Sigma\left(t_{0}\right)=\left\{x^{\mu} \mid t(x)=t_{0}\right\}
$$

are hypersurfaces of constant time. The $4 \mathrm{D}$ hypersurface $\Sigma\left(t_{0}\right) \subset \mathcal{M}$ is homeomorphic to a spacelike 3D submanifold $\hat{\Sigma}$ with coordinates $x^{i}, i=1,2,3$, and the homeomorphism forms an embedding of $\hat{\Sigma}$ into $\mathcal{M}$, which may be expressed as

$$
x_{t_{0}}^{\mu}=x^{\mu}\left(\mathbf{x}, t_{0}\right)
$$

for fixed $t_{0}$. The trajectory

$$
x_{\mathbf{x}_{0}}^{\mu}(t)=x^{\mu}\left(\mathbf{x}_{0}, t\right)
$$

associated with this embedding connects the point $\mathbf{x}_{0}$ with fixed 3D coordinates on different hyperspaces, suggesting a notion of time evolution from one hyperspace to the next.

We extend these ideas to SHP general relativity, taking advantage of the analogy with the $3+1$ formalism $[1,11,27,28]$ and employing its standard notation. Roughly following the tutorial exposition of $3+1$ numerical relativity that is given in $[29,30]$, we decompose the Einstein field equations into spacetime and $\tau$ sectors, leading to a set of coupled partial differential equations for the phase space variables of the field theory, $\gamma_{\mu v}(x, \tau)$ and $\dot{\gamma}_{\mu v}(x, \tau)=\partial \gamma_{\mu v}(x, \tau) / \partial \tau$. Although the general presentation is familiar, it differs in certain details, because the foliation is natural and the field theory is presumed to carry the factor $\sigma$ associated with objects carrying a five-index. With appropriate initial conditions for the metric and the matter distribution, this poses an initial value problem that can be integrated forward in $\tau$ to solve for evolving spacetime configurations.

\subsection{Embedding and Foliation}

The first step is to introduce a $5 \mathrm{D}$ pseudo-spacetime by defining the injective mapping

$$
\Phi: \mathcal{M} \longrightarrow \mathcal{M}_{5}=\mathcal{M} \times R \quad X=\Phi(x, \tau)=\left(x, c_{5} \tau\right)
$$

with coordinates $X^{\alpha} \in \mathcal{M}_{5}$, for $\alpha=0,1,2,3,5$. This structure admits the natural foliation defined by level surfaces of the scalar field $\tau(X)=\tau$

$$
\Sigma\left(\tau_{0}\right)=\left\{X \in \mathcal{M}_{5} \mid \tau(X)=X^{5} / c_{5}=\tau_{0}\right\}
$$

which is homeomorphic to $\mathcal{M}\left(\tau_{0}\right)$ for any $\tau_{0}$ (and so we drop reference to $\tau_{0}$ in referring to the hypersurfaces). We take

$$
E_{\mu}^{\alpha}=\left(\frac{\partial X^{\alpha}(x, \tau)}{\partial x^{\mu}}\right)_{\tau_{0}} \quad \mu=0,1,2,3
$$

as the four basis elements $E_{\mu}=\partial_{\mu}$ for $\mathcal{T}(\Sigma)$, the tangent space of $\Sigma$. Thus, when restricted to $X \in \Sigma$, the squared interval becomes

$$
\left.d X^{2}\right|_{\Sigma}=\left.g_{\alpha \beta} d X^{\alpha} d X^{\beta}\right|_{\Sigma}=g_{\alpha \beta} \frac{\partial X^{\alpha}}{\partial x^{\mu}} \frac{\partial X^{\beta}}{\partial x^{v}} d x^{\mu} d x^{\nu}=\gamma_{\mu \nu} d x^{\mu} d x^{\nu}
$$


where we identify $\gamma_{\mu \nu}=g_{\alpha \beta} E_{\mu}^{\alpha} E_{v}^{\beta}$, the induced metric on $\Sigma$, with the $4 \mathrm{D}$ spacetime metric we began with. For a vector in the time direction of $\mathcal{T}\left(\mathcal{M}_{5}\right)$, we write

$$
\partial_{\alpha} \tau(X)=\delta_{\alpha}^{5} \partial_{5} \tau(X)=\delta_{\alpha}^{5} \frac{1}{c_{5}} \partial_{\tau} \tau(X)
$$

which is normal to the tangent space of $\Sigma$ in the sense that $\tau(X)=\tau_{0}$ is constant throughout $\Sigma\left(\tau_{0}\right)$. Thus, in $\mathcal{T}\left(\mathcal{M}_{5}\right)$, the vector $\left(E_{5}\right)_{\alpha}=\partial_{\alpha} \tau(X)$ points out of $\mathcal{T}(\Sigma)$ in the direction of time evolution. The unit normal $n_{\alpha}$ in the time direction is defined as

$$
n=\sigma \frac{1}{\sqrt{\left|g^{55}\right|}} E_{5} \longrightarrow n^{2}=\frac{1}{\left|g^{55}\right|} g^{\alpha \beta}\left(E_{5}\right)_{\alpha}\left(E_{5}\right)_{\beta}=\frac{1}{\left|g^{55}\right|} g^{55}=\sigma
$$

so that

$$
n^{\alpha}=g^{\alpha \beta} n_{\beta}=g^{\alpha \beta} \sigma \frac{1}{\sqrt{\mid g^{55 \mid}}} \delta_{\beta}^{5}=\sigma g^{\alpha 5} \frac{1}{\sqrt{\mid g^{55 \mid}}} .
$$

For any vector $A \in \mathcal{T}\left(\mathcal{M}_{5}\right)$ in the tangent space of $\mathcal{M}_{5}$ we can project onto parallel and normal components

$$
A_{\|}=\sigma(A \cdot n) n \quad A_{\perp}=A-\sigma(A \cdot n) n
$$

and so define the normal projection operator

$$
\Pi_{\alpha \beta}=\sigma n_{\alpha} n_{\beta} \quad \Pi_{\alpha \gamma} \Pi^{\gamma \beta}=\sigma^{2} n^{2} n_{\alpha} n^{\beta}=\Pi_{\alpha}^{\beta}
$$

and the tangent projection operator

$$
P_{\alpha \beta}=g_{\alpha \beta}-\sigma n_{\alpha} n_{\beta} \quad P^{\alpha \beta}=g^{\alpha \beta}-\sigma n^{\alpha} n^{\beta} \quad P_{\alpha \gamma} P^{\gamma \beta}=P_{\alpha}^{\beta}=\delta_{\alpha}^{\beta}-\sigma n_{\alpha} n^{\beta}
$$

along with the completeness relation

$$
g_{\alpha \beta}=P_{\alpha \beta}+\sigma n_{\alpha} n_{\beta} \quad \delta_{\beta}^{\alpha}=P_{\beta}^{\alpha}+\sigma n^{\alpha} n_{\beta} .
$$

For any vector $V \in \mathcal{T}\left(\mathcal{M}_{5}\right)$, the vector $V_{\perp}^{\alpha}=P_{\beta}^{\alpha} V^{\beta}$ is in $\mathcal{T}(\Sigma)$, and so there is some vector $v \in \mathcal{T}(\mathcal{M})$, such that

$$
V_{\perp}^{\alpha}=v^{\mu} E_{\mu}^{\alpha}
$$

which entails

$$
v_{\mu}=\gamma_{\mu \nu} v^{v}=g_{\alpha \beta} E_{\mu}^{\alpha} E_{v}^{\beta} v^{v}=g_{\alpha \beta} E_{\mu}^{\alpha} V_{\perp}^{\beta}=E_{\mu}^{\alpha} V_{\alpha}^{\perp}=E_{\mu}^{\alpha} P_{\alpha}^{\beta} V_{\beta}=E_{\mu}^{\beta} V_{\beta}
$$

since $E_{\mu}^{\alpha} \in \mathcal{T}(\Sigma)$. In particular, expressing the metric in terms of (80), we find

$$
\gamma_{\mu v}=g_{\alpha \beta} E_{\mu}^{\alpha} E_{v}^{\beta}=\left(P_{\alpha \beta}+\sigma n_{\alpha} n_{\beta}\right) E_{\mu}^{\alpha} E_{v}^{\beta}=P_{\alpha \beta} E_{\mu}^{\alpha} E_{v}^{\beta}=P_{\mu v}
$$

so that the projector $P_{\alpha \beta}$ when restricted to $\Sigma$ acts precisely as the $4 \mathrm{D}$ metric $\gamma_{\mu v}$.

Generalizing the characterization of 5D distance that is expressed in (19), we write

$$
X_{2}-X_{1}=\left(\delta x^{\mu}+N^{\mu} \delta x^{5}, N \delta x^{5}\right) \quad \delta X^{\alpha}=\left(\delta x^{\mu}+N^{\mu} \delta x^{5}\right) E_{\mu}^{\alpha}+N n^{\alpha} \delta x^{5}
$$

where $N$ is a lapse function and $N^{\mu}$ is a shift four-vector. The 5D squared invariant interval now takes the form 


$$
\begin{aligned}
d X^{2} & =g_{\alpha \beta}(x, \tau) \delta X^{\alpha} \delta X^{\beta} \\
& =g_{\alpha \beta}(x, \tau)\left[\left(\delta x^{\mu}+N^{\mu} \delta x^{5}\right) E_{\mu}^{\alpha}+N n^{\alpha} \delta x^{5}\right]\left[\left(\delta x^{v}+N^{v} \delta x^{5}\right) E_{v}^{\beta}+N n^{\beta} \delta x^{5}\right] \\
& =\gamma_{\mu v}(x, \tau)\left(\delta x^{\mu}+N^{\mu} \delta x^{5}\right)\left(\delta x^{v}+N^{v} \delta x^{5}\right)+\sigma^{2} N^{2}\left(\delta x^{5}\right)^{2} \\
& =\gamma_{\mu v}(x, \tau) \delta x^{\mu} \delta x^{v}+2 \gamma_{\mu v}(x, \tau) N^{v} \delta x^{\mu} \delta x^{5}+\left(\gamma_{\mu v}(x, \tau) N^{\mu} N^{v}+\sigma^{2} N^{2}\right)\left(\delta x^{5}\right)^{2}
\end{aligned}
$$

allowing us to decompose the 5D metric

$$
g_{\alpha \beta}=\left[\begin{array}{cc}
\gamma_{\mu \nu} & N_{\mu} \\
N_{\mu} & \sigma N^{2}+\gamma_{\mu \nu} N^{\mu} N^{v}
\end{array}\right] \quad g^{\alpha \beta}=\left[\begin{array}{cc}
\gamma^{\mu \nu}+\sigma \frac{1}{N^{2}} N^{\mu} N^{v} & -\sigma \frac{1}{N^{2}} N^{\mu} \\
-\sigma \frac{1}{N^{2}} N^{\mu} & \sigma \frac{1}{N^{2}}
\end{array}\right]
$$

into the spacetime and $\tau$ sectors. Once again, on any $4 \mathrm{D}$ SHP spacetime $\mathcal{M}(\tau)$, the induced metric $\gamma_{\mu \nu}(x, \tau)$ is just the local metric, we assumed to exist at the outset. In this decomposition, the unit normal $n^{\alpha}$ becomes

$$
n_{\alpha}=\sigma \frac{1}{\sqrt{\left|g^{55}\right|}} \partial_{\alpha} \tau(X)=\sigma N \delta_{\alpha}^{5} .
$$

One can easily establish that $\sqrt{g}=\sqrt{\gamma} N$ by writing

$$
\left[\begin{array}{ll}
g_{\mu \nu} & g_{\mu 5} \\
g_{\mu 5} & g_{55}
\end{array}\right]=\left[\begin{array}{cc}
\gamma_{\mu v} & N_{\mu} \\
N_{\mu} & \sigma N^{2}+\gamma_{\mu v} N^{\mu} N^{v}
\end{array}\right]=\left[\begin{array}{cc}
I & 0 \\
N^{\mu} & 1
\end{array}\right]\left[\begin{array}{cc}
\gamma_{\mu v} & 0 \\
0 & \sigma N^{2}
\end{array}\right]\left[\begin{array}{cc}
I & N^{v} \\
0 & 1
\end{array}\right] .
$$

\subsection{Intrinsic and Extrinsic Geometry}

With compatible connection (24) the covariant derivative (45) on $\mathcal{M}_{5}$ obeys $\nabla_{\gamma} g_{\alpha \beta}=0$, leading to the standard Ricci identity

$$
\left[\nabla_{\beta}, \nabla_{\alpha}\right] X_{\delta}=X_{\gamma} R_{\delta \alpha \beta}^{\gamma}
$$

with Riemann tensor

$$
R_{\delta \alpha \beta}^{\gamma}=\frac{\partial}{\partial x^{\alpha}} \Gamma_{\delta \beta}^{\gamma}-\frac{\partial}{\partial x^{\beta}} \Gamma_{\delta \alpha}^{\gamma}+\Gamma_{\sigma \alpha}^{\gamma} \Gamma_{\delta \beta}^{\sigma}-\Gamma_{\sigma \beta}^{\gamma} \Gamma_{\delta \alpha}^{\sigma}
$$

and associated Bianchi relations. To find the corresponding structures on the hyperspaces defined through foliation we examine their projections onto $\mathcal{T}(\Sigma)$.

For a vector $V=V^{\perp} \in \mathcal{T}(\Sigma)$ we define the projected covariant derivative $\bar{\nabla}_{\alpha}$ in which the projected derivative acts on the projected vector. Thus,

$$
\bar{\nabla}_{\alpha} V_{\beta}^{\perp}=\bar{\nabla}_{\alpha}\left(P_{\beta}^{\delta} V_{\delta}\right)=P_{\beta}^{\delta} \bar{\nabla}_{\alpha}\left(V_{\delta}\right)=P_{\beta}^{\delta}\left(P_{\alpha}^{\gamma} \nabla_{\gamma}\right) V_{\delta}=P_{\alpha}^{\gamma} P_{\beta}^{\delta} \nabla_{\gamma} V_{\delta} .
$$

We justify the second equality by noting that the full $5 \mathrm{D}$ covariant derivative of the projector is

$$
\nabla_{\alpha} P_{\beta \gamma}=\nabla_{\alpha}\left(g_{\beta \gamma}-\sigma n_{\beta} n_{\gamma}\right)=-\sigma \nabla_{\alpha}\left(n_{\beta} n_{\gamma}\right)=-\sigma\left[\left(\nabla_{\alpha} n_{\gamma}\right) n_{\beta}+n_{\beta} \nabla_{\alpha} n_{\gamma}\right]
$$

and so the projected covariant derivative of the projector is

$$
\begin{aligned}
\bar{\nabla}_{\alpha} P_{\beta \gamma} & =-\sigma P_{\alpha}^{\alpha^{\prime}} P_{\beta}^{\beta^{\prime}} P_{\gamma}^{\gamma^{\prime}}\left(\left(\nabla_{\alpha^{\prime}} n_{\gamma^{\prime}}\right) n_{\beta^{\prime}}+n_{\beta^{\prime}} \nabla_{\alpha^{\prime}} n_{\gamma^{\prime}}\right) \\
& =-\sigma P_{\alpha}^{\alpha^{\prime}} P_{\gamma}^{\gamma^{\prime}}\left(\nabla_{\alpha^{\prime}} n_{\gamma^{\prime}}\right)\left(P_{\beta}^{\beta^{\prime}} n_{\beta^{\prime}}\right)-\sigma P_{\alpha}^{\alpha^{\prime}} P_{\gamma}^{\gamma^{\prime}}\left(P_{\beta}^{\beta^{\prime}} n_{\beta^{\prime}}\right) \nabla_{\alpha^{\prime}} n_{\gamma^{\prime}}=0
\end{aligned}
$$


which follows from $P_{\beta}^{\delta} n_{\delta} \equiv 0$. This compatibility justifies regarding $\bar{\nabla}_{\alpha}$ as the intrinsic covariant derivative on $\mathcal{T}(\Sigma)$, denoted as

$$
D_{\alpha}=\bar{\nabla}_{\alpha}=P_{\alpha}^{\gamma} \nabla_{\gamma} \quad D_{\mu}=E_{\mu}^{\alpha} D_{\alpha}=E_{\mu}^{\alpha} P_{\alpha}^{\gamma} \nabla_{\gamma}=E_{\mu}^{\gamma} \nabla_{\gamma}
$$

and satisfying $D_{\mu} \gamma_{\lambda \rho}=0$. That is, for $V_{\mu}^{\perp} \in \mathcal{T}(\Sigma)$ and $v_{\nu} \in \mathcal{T}(\mathcal{M})$ with $v_{\mu}=E_{\mu}^{\alpha} V_{\alpha}^{\perp}$ we have

$$
E_{\mu}^{\alpha} E_{v}^{\beta}\left(D_{\alpha} V_{\beta}^{\perp}\right)=E_{\mu}^{\alpha} D_{\alpha} E_{v}^{\beta}\left(P_{\beta}^{\delta} V_{\delta}\right)=E_{\mu}^{\alpha} D_{\alpha}\left(E_{v}^{\beta} P_{\beta}^{\delta}\right) V_{\delta}=E_{\mu}^{\alpha} D_{\alpha} E_{v}^{\delta} V_{\delta}=D_{\mu} v_{v}
$$

The projected curvature $\bar{R}_{\lambda \mu v}^{\rho}$ is defined through

$$
\left[D_{v}, D_{\mu}\right] X_{\lambda}=X_{\rho} \bar{R}_{\lambda \mu v}^{\rho}
$$

and will be examined below.

Restricted to $\mathcal{T}(\Sigma) \subset \mathcal{T}(\mathcal{M})$ the Weingarten map $\chi$ associates to a tangent vector $V \in \mathcal{T}(\Sigma)$ the variation of the $\tau$-like unit vector $n$ along $V$. Thus,

$$
\chi(V)=\nabla_{V} n=V \cdot(\nabla n) \quad \chi^{\alpha}(V)=V^{\beta} \nabla_{\beta} n^{\alpha}
$$

and

$$
U \cdot\left(\nabla_{V} n\right)=V \cdot\left(\nabla_{U} n\right)
$$

The extrinsic curvature on $\mathcal{T}(\Sigma)$ is

$$
K: \mathcal{T}(\Sigma) \times \mathcal{T}(\Sigma) \rightarrow R
$$

defined as the projection onto a vector $U$ of the Weingarten map along a vector $V$

$$
\begin{aligned}
K(U, V) & =-U \cdot \chi(V)=-U \cdot \nabla_{V} n=-g_{\alpha \gamma} V^{\alpha} U^{\beta} \nabla_{\beta} n^{\gamma} \\
K_{\alpha \beta} & =-g_{\alpha \gamma} \nabla_{\beta} n^{\gamma}=-\nabla_{\beta} n_{\alpha} .
\end{aligned}
$$

Using the projector $P_{\alpha \beta}$, we extend this definition to the full manifold $\mathcal{T}(\mathcal{M})$ as

$$
\begin{aligned}
K\left(U_{\perp}, V_{\perp}\right) & =K(P U, P V)=-g_{\gamma \alpha}\left(P_{\varepsilon}^{\gamma} V^{\varepsilon}\right)\left(P_{\phi}^{\beta} U^{\phi}\right) \nabla_{\beta} n^{\alpha} \\
K_{\phi \varepsilon} U^{\phi} V^{\varepsilon} & =V^{\varepsilon} U^{\phi}\left(-g_{\gamma \alpha} P_{\varepsilon}^{\gamma} P_{\phi}^{\beta}\right) \nabla_{\beta} n^{\alpha} \\
K_{\alpha \beta} & =-P_{\alpha}^{\gamma} P_{\beta}^{\delta} \nabla_{\delta} n_{\gamma}
\end{aligned}
$$

where we recall that $\nabla_{\delta} n_{\gamma}$ may have both normal and tangent components with respect to $\mathcal{T}(\Sigma)$. Because the projection is idempotent, we can write

$$
P_{\beta}^{\delta}\left(\nabla_{\gamma} n_{\delta}\right) \equiv \nabla_{\gamma} n_{\beta}
$$

leading to the identity

$$
K_{\alpha \beta}=-P_{\alpha}^{\gamma} P_{\beta}^{\delta} \nabla_{\gamma} n_{\delta}=-P_{\alpha}^{\gamma} \nabla_{\gamma} n_{\beta}=-\left(\gamma_{\alpha}^{\gamma}-\sigma n_{\alpha} n^{\gamma}\right) \nabla_{\gamma} n_{\beta}=-\nabla_{\alpha} n_{\beta}+\sigma n_{\alpha}\left(n^{\gamma} \nabla_{\gamma} n_{\beta}\right)
$$

and the contracted form

$$
K=\gamma^{\alpha \beta} K_{\alpha \beta}=\gamma^{\alpha \beta} P_{\alpha}^{\gamma} P_{\beta}^{\delta} \nabla_{\gamma} n_{\delta}=\gamma^{\gamma \delta} \nabla_{\gamma} n_{\delta}=\nabla_{\alpha} n^{\alpha}
$$


Using (87) for the unit normal $n_{\alpha}$, we may expand

$$
\begin{aligned}
\left(n^{\gamma} \nabla_{\gamma} n_{\beta}\right) & =\sigma n^{\gamma} \nabla_{\gamma}\left(N \nabla_{\beta} \tau\right)=\sigma n^{\gamma}\left(\nabla_{\gamma} N\right) \frac{n_{\beta}}{\sigma N}+\sigma n^{\gamma} N \nabla_{\beta}\left(\frac{n_{\gamma}}{\sigma N}\right) \\
& =\frac{1}{N}\left[n^{\gamma} n_{\beta} \nabla_{\gamma} N-\sigma \delta_{\beta}^{\gamma} \nabla_{\gamma} N\right]=-\sigma \frac{1}{N}\left[\delta_{\beta}^{\gamma}-\sigma n^{\gamma} n_{\beta}\right] \nabla_{\gamma} N \\
& =-\sigma \frac{1}{N} P_{\beta}^{\gamma} \nabla_{\gamma} N=-\sigma \frac{1}{N} D_{\beta} N
\end{aligned}
$$

to put (106) into the form

$$
K_{\alpha \beta}=-\nabla_{\alpha} n_{\beta}-n_{\alpha} \frac{1}{N} D_{\beta} N
$$

If $V \in \mathcal{T}\left(\mathcal{M}_{5}\right)$ has components both tangent and normal to $\mathcal{T}(\Sigma)$, and it so can be written as

$$
V^{\beta}=E_{\lambda}^{\beta} v^{\lambda}-\sigma(n \cdot V) n^{\beta} \longrightarrow \nabla_{\alpha} V^{\beta}=\nabla_{\alpha} E_{\lambda}^{\beta} v^{\lambda}-\sigma \nabla_{\alpha}(n \cdot V) n^{\beta}
$$

we see that

$$
D_{\mu} v_{v}=E_{\mu}^{\alpha} E_{v}^{\beta} \nabla_{\alpha} V_{\beta}-\sigma(n \cdot V) K_{\mu v}
$$

in which the first term represents the tangential part of the covariant derivative, and the second term is seen to expresses the connection for the normal components of $V$ in the full covariant derivative.

\subsection{Evolution of the Hypersurface $\Sigma$}

From (84) we see that the variation of $X \in \Sigma$ for a small time variation $\delta x^{5}$ at a given point $x_{0} \in \mathcal{M}$ is

$$
\delta X^{\alpha}=\left(\frac{\partial X^{\alpha}}{\partial x^{5}}\right)_{x_{0}} \delta x^{5}=\left(\frac{\partial X^{\alpha}}{\partial \tau}\right)_{x_{0}} \delta \tau \longrightarrow E_{5}^{\alpha}=\left(\partial_{5}\right)^{\alpha}=N n^{\alpha}+N^{\mu} E_{\mu}^{\alpha}
$$

Defining $m^{\alpha}=N n^{\alpha}$ we write $E_{5}$ as $\partial_{5}=m+\mathbf{N}$ and characterize time evolution through the Lie derivative in the time direction

$$
\mathcal{L}_{5}=\mathcal{L}_{m}+\mathcal{L}_{\mathbf{N}}
$$

For the metric $\gamma_{\alpha \beta}$, the Lie derivative is

$$
\mathcal{L}_{m} \gamma_{\alpha \beta}=m^{\gamma} \nabla_{\gamma} \gamma_{\alpha \beta}+\gamma_{\gamma \beta} \nabla_{\alpha} m^{\gamma}+\gamma_{\alpha \gamma} \nabla_{\beta} m^{\gamma}
$$

which we may evaluate by using (79) for $P_{\alpha \beta}=\gamma_{\alpha \beta}$ in the first term and using (109) to obtain

$$
\nabla_{\beta} m_{\alpha}=N \nabla_{\beta} n_{\alpha}+n_{\alpha} \nabla_{\beta} N=-N K_{\beta \alpha}-n_{\beta} D_{\alpha} N+n_{\alpha} \nabla_{\beta} N u
$$

in the remaining terms. Notice that $\mathcal{L}_{m} P_{\beta}^{\alpha}$ is the derivative in the normal direction of the projector onto the tangent space, so that direct calculation while using (109) and (115) provides

$$
\mathcal{L}_{m} P_{\beta}^{\alpha}=m^{\gamma} \nabla_{\gamma}\left(\delta_{\beta}^{\alpha}-\sigma n^{\alpha} n_{\beta}\right)-\gamma_{\beta}^{\gamma} \nabla_{\gamma} m^{\alpha}+\gamma_{\gamma}^{\alpha} \nabla_{\beta} m^{\gamma}=0
$$

expressing compatibility of $\mathcal{L}_{m}$ with $P^{\alpha}$. As a result, if $V \in \mathcal{T}\left(\mathcal{M}_{5}\right)$ is tangent to $\Sigma$, its Lie derivative in the time direction is tangent to $\Sigma$, and so tangent vectors propagate as tangent vectors as $\tau$ advances monotonically. As a result, (114) simplifies to

$$
\mathcal{L}_{m} \gamma_{\alpha \beta}=-2 N K_{\alpha \beta}
$$

leading to 


$$
\mathcal{L}_{5} \gamma_{\alpha \beta}-\mathcal{L}_{\mathbf{N}} \gamma_{\alpha \beta}=-2 N K_{\alpha \beta} \longrightarrow \mathcal{L}_{5} \gamma_{\mu \nu}-\mathcal{L}_{\mathbf{N}} \gamma_{\mu \nu}=-2 N K_{\mu \nu}
$$

as the evolution equation for the metric.

\subsection{Decomposition of the Riemann Tensor}

The $4+1$ decomposition of $R_{\delta \alpha \beta}^{\gamma}$ is accomplished by projecting onto $\Sigma$ and $n$. Using the completeness relation (80) to write

$$
R_{\delta \alpha \beta}^{\gamma}=\left(P_{\alpha}^{\alpha^{\prime}}+\sigma n_{\alpha} n^{\alpha^{\prime}}\right)\left(P_{\beta}^{\beta^{\prime}}+\sigma n_{\beta} n^{\beta^{\prime}}\right)\left(P_{\gamma^{\prime}}^{\gamma}+\sigma n^{\gamma} n_{\gamma^{\prime}}\right)\left(P_{\delta}^{\delta^{\prime}}+\sigma n_{\delta} n^{\delta^{\prime}}\right) R_{\delta^{\prime} \alpha^{\prime} \beta^{\prime}}^{\gamma^{\prime}}
$$

we obtain products of the type

$$
R_{\delta \alpha \beta}^{\gamma}=\delta_{\alpha}^{\alpha^{\prime}} \delta_{\beta}^{\beta^{\prime}} \delta_{\gamma^{\prime}}^{\gamma} \delta_{\delta}^{\delta^{\prime}} R_{\delta^{\prime} \alpha^{\prime} \beta^{\prime}}^{\gamma^{\prime}} \longrightarrow\left\{\begin{array}{l}
E_{\mu}^{\alpha} E_{v}^{\beta} E_{\gamma}^{\lambda} E_{\sigma}^{\delta} P_{\alpha}^{\alpha^{\prime}} P_{\beta}^{\beta^{\prime}} P_{\gamma^{\prime}}^{\gamma} P_{\delta}^{\delta^{\prime}} R_{\delta^{\prime} \alpha^{\prime} \beta^{\prime} \gamma^{\prime}}=R_{\sigma \mu \nu}^{\lambda} \\
E_{\mu}^{\alpha} E_{v}^{\beta} E_{\gamma}^{\lambda} P_{\gamma^{\prime}}^{\gamma} n^{\delta} P_{\alpha}^{\alpha^{\prime}} P_{\beta}^{\beta^{\prime}} R_{\delta \alpha^{\prime} \beta^{\prime}}^{\gamma^{\prime}}=\sigma N R_{5 \mu \nu}^{\lambda} \\
E^{\alpha \mu} E_{v}^{\beta} P_{\alpha \alpha^{\prime}} n^{\delta} P_{\beta}^{\beta^{\prime}} n^{\gamma} R_{\delta \beta^{\prime} \gamma}^{\alpha^{\prime}}=N^{2} R_{5 v 5}^{\mu}
\end{array}\right.
$$

where $R_{\delta \alpha \beta}^{\gamma} n^{\delta} n^{\alpha} n^{\beta}=0$, because of the symmetries of the Riemann tensor. For the projected curvature defined in (96), we write

$$
D_{\alpha} D_{\beta} V^{\gamma}=D_{\alpha}\left(D_{\beta} V^{\gamma}\right)=P_{\alpha}^{\alpha^{\prime}} P_{\beta}^{\beta^{\prime}} P_{\gamma}^{\gamma^{\prime}} \nabla_{\alpha^{\prime}}\left(D_{\beta^{\prime}} V^{\gamma^{\prime}}\right)
$$

we expand and use (92) in order to obtain

$$
D_{\alpha} D_{\beta} V^{\gamma}=\sigma K_{\alpha \beta} P_{\gamma^{\prime}}^{\gamma} n^{\beta^{\prime}} \nabla_{\beta^{\prime}} V^{\gamma^{\prime}}+\sigma K_{\alpha}^{\gamma} K_{\beta \delta} V^{\delta}+P_{\alpha}^{\alpha^{\prime}} P_{\beta}^{\beta^{\prime \prime}} P_{\gamma^{\prime \prime}}^{\gamma}\left(\nabla_{\alpha^{\prime}} \nabla_{\beta^{\prime \prime}} V^{\gamma^{\prime \prime}}\right)
$$

so that

$$
\left[D_{\alpha}, D_{\beta}\right] V^{\gamma}=\bar{R}_{\delta \alpha \beta}^{\gamma} V^{\delta}=-\sigma\left(K_{\alpha \delta} K_{\beta}^{\gamma}-K_{\beta \delta} K_{\alpha}^{\gamma}\right) V^{\delta}+P_{\alpha}^{\alpha^{\prime}} P_{\beta}^{\beta^{\prime}} P_{\gamma^{\prime}}^{\gamma}{ }_{\delta^{\prime} \alpha^{\prime} \beta^{\prime}}^{\gamma^{\prime}} P_{\delta}^{\delta^{\prime}} V^{\delta}
$$

which, by the quotient theorem on $\Sigma$, leads to

$$
P_{\alpha}^{\alpha^{\prime}} P_{\beta}^{\beta^{\prime}} P_{\gamma^{\prime}}^{\gamma}{ }^{\delta^{\prime}} R_{\delta}^{\gamma_{\delta^{\prime} \alpha^{\prime} \beta^{\prime}}^{\prime}}=\bar{R}_{\delta \alpha \beta}^{\gamma}-\sigma\left(K_{\alpha}^{\gamma} K_{\beta \delta}-K_{\beta}^{\gamma} K_{\alpha \delta}\right)
$$

This is known as the Gauss relation. Acting on this expression with $E_{\gamma}^{\mu} E_{\nu}^{\delta} E_{\lambda}^{\alpha} E_{\rho}^{\beta}$ we find

$$
R^{\mu}{ }_{\nu \lambda \rho}=\bar{R}_{v \lambda \rho}^{\mu}-\sigma\left(K_{\lambda}^{\mu} K_{\rho v}-K_{\rho}^{\mu} K_{\lambda v}\right)
$$

providing an expression for the intrinsic curvature $R_{\nu \lambda \rho}^{\mu}$ in terms of the projected curvature $\bar{R}_{\nu \lambda \rho}^{\mu}$ and the intrinsic curvature $K_{\rho v}$. Contracting on $\alpha$ and $\gamma$ in (124) leads to

$$
P_{\alpha}^{\alpha^{\prime}} P_{\beta}^{\beta^{\prime}} R_{\alpha^{\prime} \beta^{\prime}}-\sigma P_{\alpha \alpha^{\prime}} n^{\delta^{\prime}} P_{\beta}^{\beta^{\prime}} n^{\gamma^{\prime}} R_{\delta^{\prime} \beta^{\prime} \gamma^{\prime}}^{\alpha^{\prime}}=\bar{R}_{\alpha \beta}-\sigma\left(K K_{\alpha \beta}-K_{\alpha}^{\delta} K_{\beta \delta}\right)
$$

and contracting on $\alpha$ and $\beta$ gives

$$
R-2 \sigma R_{\alpha \beta} n^{\alpha} n^{\beta}=\bar{R}-\sigma\left(K^{2}-K^{\alpha \beta} K_{\alpha \beta}\right)
$$

called the scalar Gauss relation.

Applying the Ricci identity (89) to the vector $n$ as

$$
\left(\nabla_{\beta} \nabla_{\alpha}-\nabla_{\alpha} \nabla_{\beta}\right) n^{\gamma}=R_{\gamma^{\prime} \alpha \beta}^{\gamma} n^{\gamma^{\prime}}
$$

projecting the LHS onto $\Sigma$ as 


$$
P_{\alpha}^{\alpha^{\prime}} P_{\beta}^{\beta^{\prime}} P_{\gamma^{\prime}}^{\gamma}\left(\nabla_{\alpha^{\prime}} \nabla_{\beta^{\prime}}-\nabla_{\beta^{\prime}} \nabla_{\alpha^{\prime}}\right) n^{\gamma^{\prime}}
$$

and using the identity (106) leads us to

$$
D_{\beta} K_{\alpha}^{\gamma}-D_{\alpha} K_{\beta}^{\gamma}=P_{\gamma^{\prime}}^{\gamma} n^{\delta} P_{\alpha}^{\alpha^{\prime}} P_{\beta}^{\beta^{\prime}} R_{\delta \alpha^{\prime} \beta^{\prime}}^{\gamma^{\prime}}
$$

which is called the Codazzi relation. Contracting on $\alpha$ and $\gamma$ produces

$$
n_{\delta} R_{\mu \nu \lambda}^{\delta}=D_{\lambda} K_{v \mu}-D_{v} K_{\lambda \mu}
$$

Using (87) for the unit normal $n_{\alpha}$ provides an interpretation of this expression as

$$
n_{\delta} R_{\mu \nu \lambda}^{\delta}=\sigma N \delta_{\delta}^{5} R_{\mu \nu \lambda}^{\delta} \longrightarrow R_{\mu \nu \lambda}^{5}=\sigma \frac{1}{N}\left(D_{\lambda} K_{v \mu}-D_{v} K_{\lambda \mu}\right)
$$

recalling the role of the extrinsic curvature $K_{\mu \nu}$ as the curvature of $\mathcal{M}$ mapped to the hypersurface $\Sigma$ and embedded in the larger manifold $\mathcal{M}_{5}$.

Returning to the Ricci identity for $n^{\alpha}$, we apply (109) twice to terms $\nabla_{\beta} \nabla_{\gamma} n^{\alpha}$ and project onto (128) with $P_{\alpha \alpha^{\prime}}{ }^{\prime} \gamma^{\prime} P_{\beta}^{\beta^{\prime}}$ to obtain

$$
-K_{\alpha \gamma} K_{\beta}^{\gamma}+\frac{1}{N} D_{\beta} D_{\alpha} N+P_{\alpha}^{\alpha^{\prime}} P_{\beta}^{\beta^{\prime}} n^{\gamma} \nabla_{\gamma} K_{\alpha^{\prime} \beta^{\prime}}=P_{\alpha \alpha^{\prime}} n^{\gamma^{\prime}} P_{\beta}^{\beta^{\prime}} R_{\delta \beta^{\prime} \gamma^{\prime}}^{\alpha^{\prime}} n^{\delta} .
$$

Again using (115) in the Lie derivative of $K_{\alpha \beta}$ to write

$$
\mathcal{L}_{m} K_{\alpha \beta}=N n^{\gamma} \nabla_{\gamma} K_{\alpha \beta}-2 N K_{\alpha \gamma} K_{\beta}^{\gamma}-K_{\alpha \gamma} D^{\gamma} N n_{\beta}-K_{\beta \gamma} D^{\gamma} N n_{\alpha}
$$

the last two equations are combined as

$$
\frac{1}{N} \mathcal{L}_{m} K_{\alpha \beta}+\frac{1}{N} D_{\alpha} D_{\beta} N+K_{\alpha \gamma} K_{\beta}^{\gamma}=P_{\alpha \alpha^{\prime}}{ }^{\delta} P_{\beta}^{\beta^{\prime}} n^{\gamma} R_{\delta \beta^{\prime} \gamma}^{\alpha^{\prime}}
$$

to provide an evolution equation for $K_{\alpha \beta}$. Rewriting (126) as

$$
P_{\alpha \alpha^{\prime}} n^{\delta^{\prime}} P_{\beta}^{\beta^{\prime}} n^{\gamma^{\prime}} R_{\delta^{\prime} \beta^{\prime} \gamma^{\prime}}^{\alpha^{\prime}}=\sigma P_{\alpha}^{\alpha^{\prime}} P_{\beta}^{\beta^{\prime}} R_{\alpha^{\prime} \beta^{\prime}}-\sigma \bar{R}_{\alpha \beta}+K K_{\alpha \beta}-K_{\alpha}^{\delta} K_{\beta \delta}
$$

we can put (135) into the form

$$
P_{\alpha}^{\alpha^{\prime}} P_{\beta}^{\beta^{\prime}} R_{\alpha^{\prime} \beta^{\prime}}=\sigma \frac{1}{N} \mathcal{L}_{m} K_{\alpha \beta}+\sigma \frac{1}{N} D_{\alpha} D_{\beta} N+\bar{R}_{\alpha \beta}-\sigma K K_{\alpha \beta}+\sigma 2 K_{\alpha}^{\delta} K_{\beta \delta}
$$

in which only $P_{\alpha}^{\alpha^{\prime}} P_{\beta}^{\beta^{\prime}} R_{\alpha^{\prime} \beta^{\prime}}$ on the LHS refers to the $5 \mathrm{D}$ geometry of $\mathcal{M}_{5}$.

\subsection{Decomposition of the Einstein Equation}

The Einstein equations

$$
G_{\alpha \beta}=R_{\alpha \beta}-\frac{1}{2} g_{\alpha \beta} R=\frac{8 \pi G}{c^{4}} T_{\alpha \beta}
$$

can be written

$$
R_{\alpha \beta}=\frac{8 \pi G}{c^{4}}\left(T_{\alpha \beta}-\frac{1}{2} g_{\alpha \beta} T\right)
$$

where $T=g^{\alpha \beta} T_{\alpha \beta}$. As above, we decompose the field equations by projecting onto $\Sigma$ and $n$ as

$$
T_{\alpha \beta}=T_{\alpha^{\prime} \beta^{\prime}}\left(P_{\alpha}^{\alpha^{\prime}}+\sigma n^{\alpha^{\prime}} n_{\alpha}\right)\left(P_{\beta}^{\beta^{\prime}}+\sigma n^{\beta^{\prime}} n_{\beta}\right)=S_{\alpha \beta}+2 \sigma n_{\alpha} p_{\beta}+n_{\alpha} n_{\beta} \mathcal{K}
$$


where

$$
S_{\alpha \beta}=P_{\alpha}^{\alpha^{\prime}} P_{\beta}^{\beta^{\prime}} T_{\alpha^{\prime} \beta^{\prime}} \quad p_{\beta}=-n^{\alpha^{\prime}} P_{\beta}^{\beta^{\prime}} T_{\alpha^{\prime} \beta^{\prime}} \quad \quad \kappa=n^{\alpha} n^{\beta} T_{\alpha \beta}
$$

so that $S_{\mu \nu}$ corresponds to the $4 \mathrm{D}$ energy-momentum tensor $T_{\mu v}, p_{\mu}$ corresponds to the mass current into the $\mu$ direction $T_{5 \mu}$, and $\kappa$ corresponds to the mass density $T_{55}$. It is useful to regard mass in this context as being related to the difference between energy and momentum, a dynamical quantity in the SHP framework. The trace is

$$
T=g^{\alpha \beta} T_{\alpha \beta}=g^{\alpha \beta}\left(S_{\alpha \beta}-2 \sigma n_{\alpha} p_{\beta}+n_{\alpha} n_{\beta} \kappa\right)=S-2 \sigma g^{\alpha \beta} n_{\alpha} p_{\beta}+g^{\alpha \beta} n_{\alpha} n_{\beta} \kappa=S+\sigma \kappa
$$

where we used

$$
g^{\alpha \beta} n_{\alpha} p_{\beta}=n \cdot p=0
$$

which follows from

$$
p_{\beta}=-P_{\beta}^{\beta^{\prime}}\left(n^{\alpha} T_{\alpha \beta^{\prime}}\right) \in \mathcal{T}(\Sigma) .
$$

Thus, projecting the field equations (139) onto $\Sigma$ with $P_{\alpha}^{\alpha^{\prime}} P_{\beta}^{\beta^{\prime}}$ leads to

$$
P_{\alpha}^{\alpha^{\prime}} P_{\beta}^{\beta^{\prime}}\left(T_{\alpha^{\prime} \beta^{\prime}}-\frac{1}{2} g_{\alpha^{\prime} \beta^{\prime}} T\right)=S_{\alpha \beta}-\frac{1}{2} \gamma_{\alpha \beta}(S+\sigma \kappa)
$$

on the RHS and while the LHS is $P_{\alpha}^{\alpha^{\prime}} P_{\beta}^{\beta^{\prime}} R_{\alpha^{\prime} \beta^{\prime}}$ which from (137) provides

$$
\mathcal{L}_{m} K_{\mu v}=-D_{\mu} D_{v} N+N\left\{-\sigma \bar{R}_{\mu v}+K K_{\mu v}-2 K_{\mu}^{\lambda} K_{v \lambda}+\sigma \frac{8 \pi G}{c^{4}}\left[S_{\mu v}-\frac{1}{2} \gamma_{\mu v}(S+\sigma \kappa)\right]\right\}
$$

as the evolution equation for $K_{\mu v}$.

The double projection onto the time direction $n$ is

$$
\left(R_{\alpha \beta}-\frac{1}{2} g_{\alpha \beta} R\right) n^{\alpha} n^{\beta}=\frac{8 \pi G}{c^{4}} T_{\alpha \beta} n^{\alpha} n^{\beta} \quad \longrightarrow \quad R_{\alpha \beta} n^{\alpha} n^{\beta}-\frac{1}{2} \sigma R=\frac{8 \pi G}{c^{4}} \kappa
$$

and using the scalar Gauss relation (127), we obtain

$$
\bar{R}-\sigma\left(K^{2}-K^{\mu v} K_{\mu v}\right)=-\sigma \frac{16 \pi G}{c^{4}} \kappa
$$

This expression, called the Hamiltonian constraint, has no $\tau$-derivatives, and so, if it is satisfied by the initial conditions, then it will be satisfied at all times. We observe that this constraint applies to the mass density of the gravitational field, not the energy density as in 4D GR.

The mixed projection with $P_{\beta}^{\beta^{\prime}} n^{\alpha}$

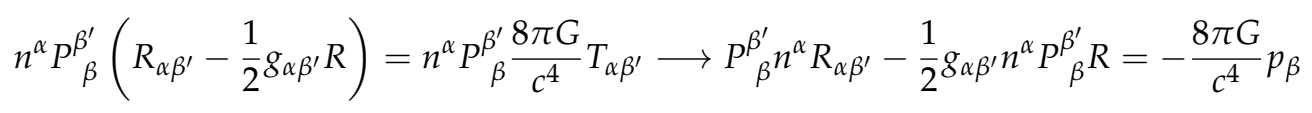

is combined with the contracted Codazzi relation (131) and $g_{\alpha \beta^{\prime}} n^{\alpha} P_{\beta}^{\beta^{\prime}}=n^{\alpha} P_{\alpha \beta}=0$ to obtain

$$
D_{\mu} K_{v}^{\mu}-D_{v} K=\frac{8 \pi G}{c^{4}} p_{v}
$$


which is called the momentum constraint, referring to the flow of mass into the field, and it also has no $\tau$-derivatives. We notice that the evolution equation contains only objects defined on $\Sigma$ and, thus, includes no factors of $\Gamma_{\mu \nu}^{5}$. Any such factors can only appear in the constraint equations.

Writing the Einstein tensor as

$$
G_{\alpha \beta}=R_{\alpha \beta}-\frac{1}{2} g_{\alpha \beta} R
$$

the Bianchi relations are

$$
\nabla_{\alpha} G^{\alpha \beta}=\partial_{\alpha} G^{\alpha \beta}+\text { Christoffel Symbols } \times G^{\alpha \beta}=0
$$

forming a set of relations among the field entities. The five equations in $5 \mathrm{D}$ reduce the number of independent components of $G^{\alpha \beta}$ from fifteen to ten, and are understood as constraints on the initial conditions of the evolution equations. Because the Einstein equations are second order in $\tau$ derivatives of the metric, a solution requires that the initial conditions include $\gamma_{\mu v}$ and $\partial_{\tau} \gamma_{\mu \nu}$ at the initial time. Expanding $\partial_{\alpha} G^{\alpha \beta}=\partial_{\mu} G^{\mu \beta}+\partial_{5} G^{5 \beta}$ to rewrite the Bianchi relations as

$$
\frac{1}{c_{5}} \partial_{\tau} G^{5 \beta}=-\partial_{\mu} G^{\mu \beta}-\text { Christoffel Symbols } \times G^{\alpha \beta}
$$

and noticing that the LHS cannot be more than second order in $\partial_{\tau}$, we see that $G^{5 \beta}$ cannot be more than first order in $\partial_{\tau}$. Therefore, the expressions contained in $G^{5 \beta}$ must be part of the initial conditions. The Einstein equations

$$
G^{\alpha \beta}=8 \pi G_{N} T^{\alpha \beta}
$$

thus split into components

$$
\begin{aligned}
G^{\mu \beta} & =0 \longrightarrow \text { ten equations of second order in } \partial_{\tau} \\
G^{5 \beta} & =0 \longrightarrow \text { five relations among the initial conditions of first order } \partial_{\tau} .
\end{aligned}
$$

Moreover, the constraints are propagated to future times because

$$
\left.G^{5 \beta}\right|_{\tau_{0}}=\left.0 \Rightarrow \partial_{\beta} G^{5 \beta}\right|_{\tau_{0}}=\left.0 \Rightarrow \partial_{\tau} G^{5 \beta}\right|_{\tau_{0}}=0
$$

and so they do not change.

\subsection{Summary of Einstein System as Differential Equations}

The decomposition of the Einstein equations into a $4+1$ system of partial differential equations permits particular structures to be solved as an initial value problem. The initial conditions that are to be specified at some $\tau$ are the metric $\gamma_{\mu \nu}$ and its first Lie derivative $K_{\mu v}$, along with the mass-energy-momentum distribution of matter as represented by $S_{\mu v}, p_{v}$, and $\kappa$. The initial conditions must satisfy the Hamiltonian constraint, a constraint on mass rather than energy,

$$
\bar{R}-\sigma\left(K^{2}-K^{\mu v} K_{\mu \nu}\right)=-\sigma \frac{16 \pi G}{c^{4}} \kappa
$$

and the momentum constraint

$$
D_{\mu} K_{v}^{\mu}-D_{v} K=\frac{8 \pi G}{c^{4}} p_{v}
$$

at the initial time. Because these constraints contain no $\tau$ derivatives, they are guaranteed to be satisfied at subsequent times. Given appropriate initial conditions, the metric is found at subsequent times by integrating forward—analytically or numerically—the coupled evolution equations 


$$
\frac{1}{c_{5}} \mathcal{L}_{\tau} \gamma_{\mu v}-\mathcal{L}_{\mathbf{N}} \gamma_{\mu v}=-2 N K_{\mu v}
$$

and

$$
\begin{aligned}
\left(\frac{1}{c_{5}} \mathcal{L}_{\tau}-\mathcal{L}_{\mathbf{N}}\right) & K_{\mu \nu}=-D_{\mu} D_{v} N \\
& +N\left\{-\sigma \bar{R}_{\mu \nu}+K K_{\mu \nu}-2 K_{\mu}^{\lambda} K_{\nu \lambda}+\sigma \frac{8 \pi G}{c^{4}}\left[S_{\mu \nu}-\frac{1}{2} \gamma_{\mu \nu}(S+\sigma \kappa)\right]\right\}
\end{aligned}
$$

We note that the lapse $N$ and shift $N^{\mu}$ are not dynamical variables, but they are part of the metric specified at the initial time.

\section{The ADM Hamiltonian Formulation}

The configuration space variable in the ADM formalism is $g_{\alpha \beta}=g_{\alpha \beta}\left(\gamma_{\mu \nu}, N^{\mu}, N\right)$, the full metric on $\mathcal{M}_{5}$. Becausre $N$ and $N^{\mu}$ are not dynamical, the phase space consists of $\gamma_{\mu v}$ and $\dot{\gamma}_{\mu v}$, as given by

$$
\dot{\gamma}_{\mu v}=\frac{1}{c_{5}} \mathcal{L}_{\tau} \gamma_{\mu \nu}=\mathcal{L}_{\mathbf{N}} \gamma_{\mu \nu}+2 N K_{\mu \nu}
$$

where we use (160) with the sign convention for $K_{\mu v}$ reversed. Contracting on $\alpha$ and $\beta$ in (137) and combining with the scalar Gauss relation (127), we obtain

$$
R=\bar{R}-\sigma\left(K^{2}-K^{\alpha \beta} K_{\alpha \beta}\right)+2 \sigma \nabla_{\alpha}\left(n^{\beta} \nabla_{\beta} n^{\alpha}-n^{\alpha} \nabla_{\beta} n^{\beta}\right)
$$

so that the Einstein-Hilbert action for GR in the absence of matter can be expanded as

$$
S_{A D M}\left[\gamma_{\mu v}, \dot{\gamma}_{\mu v}, N^{\mu}, N\right]=\int d \tau d^{4} x \sqrt{g} \bar{R}=\int d \tau d^{4} x \sqrt{\gamma} N\left[\bar{R}-\sigma\left(K^{\mu v} K_{\mu v}-K^{2}\right)\right]
$$

where $g=\left|\operatorname{det} g_{\alpha \beta}\right|$ and $\gamma=\left|\operatorname{det} \gamma_{\mu v}\right|$ and the total gradient in (163) is discarded as a boundary term. The DeWitt metric is defined as

$$
G^{\mu \nu \lambda \rho}=\frac{1}{2}\left(\gamma^{\mu \lambda} \gamma^{\nu \rho}+\gamma^{\mu \rho} \gamma^{\nu \lambda}-2 \gamma^{\mu \nu} \gamma^{\lambda \rho}\right)
$$

with inverse in $D$ dimensions

$$
G_{\mu \nu \lambda \rho}=\frac{1}{2}\left(\gamma_{\lambda \zeta} \gamma_{\rho \kappa}+\gamma_{\lambda \kappa} \gamma_{\rho \zeta}-\frac{2}{D-1} \gamma_{\lambda \rho} \gamma_{\zeta \kappa}\right)
$$

in terms of which

$$
G^{\mu \nu \lambda \rho} K_{\mu v} K_{\lambda \rho}=\frac{1}{2}\left(\gamma^{\mu \lambda} \gamma^{\nu \rho}+\gamma^{\mu \rho} \gamma^{\nu \lambda}-2 \gamma^{\mu \nu} \gamma^{\lambda \rho}\right) K_{\mu v} K_{\lambda \rho}=K^{\mu \nu} K_{\mu \nu}-K^{2}
$$

so that

$$
\mathcal{L}_{A D M}\left[\gamma_{\mu \nu}, \dot{\gamma}_{\mu \nu}, N^{\mu}, N\right]=\sqrt{\gamma} N\left[-\sigma G^{\mu \nu \lambda \rho} K_{\mu v} K_{\lambda \rho}+\bar{R}\right]
$$

Because $K^{\mu v}$ is first order in derivatives, the first term has the form of kinetic energy. The canonical conjugate momentum to $\gamma_{\mu \nu}$ is

$$
\pi^{\mu \nu}=\frac{\partial \mathcal{L}_{A D M}}{\partial \dot{\gamma}_{\mu \nu}}=-2 \sigma \sqrt{\gamma} N G^{\zeta \kappa \lambda \rho} K_{\lambda \rho} \frac{\partial K_{\zeta \kappa}}{\partial \dot{\gamma}_{\mu \nu}}
$$

so that using (162) to obtain 


$$
\frac{\partial K_{\zeta \kappa}}{\partial \dot{\gamma}_{\mu \nu}}=\frac{1}{2 N} \delta_{\zeta}^{\mu} \delta_{\kappa}^{v}
$$

we find

$$
\pi^{\mu \nu}=-2 \sigma \sqrt{\gamma} N G^{\zeta \kappa \lambda \rho} K_{\lambda \rho} \frac{1}{2 N} \delta_{\zeta}^{\mu} \delta_{\kappa}^{v}=-\sigma \sqrt{\gamma} G^{\mu \nu \lambda \rho} K_{\lambda \rho}=-\sigma \sqrt{\gamma}\left(K^{\mu \nu}-\gamma^{\mu \nu} K\right)
$$

with trace

$$
\pi=\gamma_{\mu \nu} \pi^{\mu \nu}=\sigma(D-1) \sqrt{\gamma} K \longrightarrow K=\frac{\sigma}{(D-1) \sqrt{\gamma}} \pi
$$

Writing $K^{\mu v}$ in terms of $\pi^{\mu v}$

$$
K^{\mu \nu}=-\frac{\sigma}{\sqrt{\gamma}}\left(\pi^{\mu \nu}-\gamma^{\mu \nu} \frac{1}{(D-1)} \pi\right)
$$

and lowering the indices of $\pi^{\mu v}$

$$
G_{\mu \nu \lambda \rho} \pi^{\lambda \rho}=\pi_{\mu \nu}-\frac{1}{D-1} \gamma_{\mu \nu} \pi=-\sigma \sqrt{\gamma} K_{\mu \nu}
$$

we see that $K_{\mu v}$ represents the momentum conjugate to $\gamma_{\mu v}$. Replacing $K_{\mu v}$ in (162), we can write the velocity as

$$
\dot{\gamma}_{\mu \nu}=-\sigma \frac{2 N}{\sqrt{\gamma}} G_{\mu \nu \lambda \rho} \pi^{\lambda \rho}+L_{N} \gamma_{\mu \nu}
$$

in terms of the momentum and configuration variable.

Because $\bar{R}$ is independent of the lapse $N$ and shift $N^{\mu}$ the Lagrangian $\mathcal{L}_{A D M}\left[\gamma_{\mu \nu}, \dot{\gamma}_{\mu \nu}, N^{\mu}, N\right]$ contains no derivatives of $N, N^{\mu}$ and these act as Lagrange multipliers enforcing as constraints their conjugates. Thus

$$
p_{N}=\frac{\partial \mathcal{L}_{A D M}}{\partial \dot{N}} \quad p_{N^{\mu}}=\frac{\partial \mathcal{L}_{A D M}}{\partial \dot{N}^{\mu}}=0
$$

and variation with respect to the lapse and shift produces

$$
0=-\frac{\partial}{\partial N}\left(\sqrt{\gamma} N\left[R-\sigma G^{\mu \nu \lambda \rho} K_{\mu \nu} K_{\lambda \rho}\right]\right)=-\sqrt{\gamma} R+\sigma \sqrt{\gamma} G^{\mu \nu \lambda \rho} \frac{\partial}{\partial N}\left(N K_{\mu \nu} K_{\lambda \rho}\right) .
$$

Rewriting (162) as

$$
K_{\mu v}=\frac{1}{2 N}\left(\dot{\gamma}_{\mu v}-D_{\mu} N_{v}+D_{v} N_{\mu}\right) \longrightarrow N K_{\mu v} K_{\lambda \rho} \sim \frac{1}{N}
$$

the Hamiltonian constraint becomes

$$
0=\sqrt{\gamma}\left[-\sigma G^{\mu \nu \lambda \rho} K_{\mu \nu} K_{\lambda \rho}-\bar{R}\right]=\sqrt{\gamma}\left[-\sigma\left(K^{\mu \nu} K_{\mu \nu}-K^{2}\right)-\bar{R}\right]=\mathcal{H}
$$

where we used (167) and the momentum constraint is

$$
0=-\frac{\partial \mathcal{L}_{A D M}}{\partial N_{\mu}}=2 \sigma \sqrt{\gamma} D_{\nu}\left(G^{\nu \mu \lambda \rho} K_{\lambda \rho}\right)=2 \sigma \sqrt{\gamma}\left(D_{v} K^{\nu \mu}-D^{\mu} K\right)=\mathcal{H}^{\mu} .
$$

Comparison with (158) and (159) shows that these constraints correspond to the non-evolving $G_{\mu 5}$ components of the Einstein field equations. Using (171), we can also write

$$
\mathcal{H}^{v}=2 \sigma \sqrt{\gamma} D_{\mu}\left(G^{\mu \nu \lambda \rho} K_{\lambda \rho}\right)=\sigma 2 D_{\mu} \pi^{\mu \nu} .
$$


The Legendre transformation to the Hamiltonian density is

$$
\begin{aligned}
\mathcal{H}_{A D M} & =\pi^{\mu v} \dot{\gamma}_{\mu v}-\mathcal{L}_{A D M}\left[\gamma_{\mu \nu}, \dot{\gamma}_{\mu v}, N^{\mu}, N\right] \\
& =-\sigma N \sqrt{\gamma} G^{\mu \nu \lambda \rho} K_{\mu v} K_{\lambda \rho}+2 \pi^{\mu v} D_{\mu} N_{v}-\sqrt{\gamma} N R
\end{aligned}
$$

where $N, N^{\mu}$ are Lagrange multipliers and do not require kinetic terms. Integrating by parts and discarding the total gradient provides

$$
2 \pi^{\mu v} D_{\mu} N_{v}=2 D_{\mu}\left(\pi^{\mu v} N_{v}\right)-N_{v}\left(2 D_{\mu} \pi^{\mu v}\right)=N_{v} \mathcal{H}^{v}
$$

and using (179), we arrive at

$$
\mathcal{H}_{A D M}=N \mathcal{H}+N_{\nu} \mathcal{H}^{v} .
$$

Writing the Hamiltonian in the form

$$
\mathcal{H}_{A D M}=\pi^{\mu v} \dot{\gamma}_{\mu \nu}+\dot{p}_{N} \mathcal{H}+\dot{p}_{N^{\mu}} \mathcal{H}^{\mu}-\mathcal{L}_{A D M}
$$

the Hamiltonian and momentum constraints $\mathcal{H}=0$ and $\mathcal{H}^{v}=0$ are seen to be secondary constraints arising from the requirement that the primary constraints $p_{N}=0$ and $p_{N^{\mu}}=0$ are preserved under time evolution,

$$
\dot{p}_{N}=\left\{p_{N}, \mathcal{H}_{A D M}\right\}=0 \quad \dot{p}_{N^{\mu}}=\left\{p_{N^{\mu}}, \mathcal{H}_{A D M}\right\}=0 .
$$

The remaining Einstein equations-the evolution equations $G^{\mu v}=0$ - then follow from

$$
\dot{\gamma}_{\mu v}=\left\{\gamma_{\mu \nu}, \mathcal{H}_{A D M}\right\} \quad \dot{\pi}^{\mu \nu}=\left\{\pi^{\mu \nu}, \mathcal{H}_{A D M}\right\}
$$

for the canonical variables

$$
\left\{\gamma_{\mu v}, \pi^{\lambda \rho}\right\}=\frac{1}{2}\left(\delta_{\mu}^{\lambda} \delta_{v}^{\rho}+\delta_{\mu}^{\rho} \delta_{v}^{\lambda}\right)
$$

The equation for $\dot{\gamma}_{\mu \nu}$ reproduces the definition of $\pi^{\mu \nu}$, since $R$ does not contain $\dot{\gamma}_{\mu v}$ and so $\left\{\gamma_{\mu v}, R\right\}=0$. The equation for $\dot{\pi}^{\mu v}$ is thus equivalent to $G^{\mu v}=0$.

\section{Perturbations to Schwarzschild Geometry}

To get a feel for some simple possibilities in this formalism, we pose a Schwarzschild-like interval in an empty pseudo-spacetime $\mathcal{M}_{5}$

$$
d s^{2}=-c^{2} B d t^{2}+A d r^{2}+r^{2} d \theta^{2}+r^{2} \sin ^{2} \theta d \phi^{2}+\sigma N^{2} c_{5}^{2} d \tau^{2} \quad T_{\alpha \beta}=0
$$

where $N=N(x, \tau)$ and we allow the mass parameter $M=M(\tau)$ in the coefficients

$$
B(r, \tau)=A^{-1}(r, \tau)=\left(1-\frac{2 G M(\tau)}{r c^{2}}\right) \quad N^{2}=N^{2}(t, r, \tau)
$$

to be $\tau$-dependent. Although the $4 \mathrm{D}$ connection and curvature on $\mathcal{M}$ may now depend on $\tau$, these generalizations do not change their structure. The $4+1$ metric is

$$
\gamma_{\mu \nu}=\operatorname{diag}\left(-B, A, r^{2}, r^{2} \sin ^{2} \theta\right) \quad g_{5 \mu}=N_{\mu}=0 \quad g_{55}=\sigma N^{2}
$$

and so the Einstein equations reduce to

$$
\partial_{\tau} \gamma_{\mu v}=-2 N K_{\mu v} \quad \partial_{5} K_{\mu v}=-D_{\mu} D_{v} N+N\left(-\sigma \bar{R}_{\mu v}+K K_{\mu v}-2 K_{\mu}^{\lambda} K_{v \lambda}\right)
$$


with constraints

$$
\bar{R}-\sigma\left(K^{2}-K^{\mu v} K_{\mu \nu}\right)=0 \quad D_{\mu} K_{v}^{\mu}-D_{v} K=0
$$

\subsection{Constant Mass Source}

Taking $M(\tau)=m=$ constant, we find

$$
\partial_{5} \gamma_{\mu \nu}=0=-2 N K_{\mu \nu} \longrightarrow K_{\mu v}=0 \longrightarrow \bar{R}_{\mu v}=-\sigma \frac{1}{N} D_{\mu} D_{v} N
$$

for the dynamical equations, as expected for a $\tau$-independent $4 \mathrm{D}$ Schwarzschild geometry. The momentum constraint is trivially satisfied, and the Hamiltonian constraint reduces to $\bar{R}=0$. Therefore, we must have

$$
\bar{R}=\gamma^{\mu v} \bar{R}_{\mu v}=-\sigma \frac{1}{N} \gamma^{\mu v} D_{\mu} D_{v} N=0 \longrightarrow \gamma^{\mu v} D_{\mu} D_{v} N=0
$$

meaning that $N$ can be any solution to the source-free $4 \mathrm{D}$ wave equation, which in Schwarzschild geometry is

$$
\left[\partial_{0}^{2}-\frac{B}{r^{2}} \partial_{r}\left(r^{2} B \partial_{r}\right)\right] N(t, r, \tau)=0
$$

where $B$ is given in (190). Writing the Lagrangian for a test particle as

$$
L=\frac{1}{2} M g_{\alpha \beta} \dot{x}^{\alpha} \dot{x}^{\beta}=\frac{1}{2} M\left[-c^{2} B(r, \tau) \dot{t}^{2}+A(r, \tau) d r^{2}+r^{2} \dot{\theta}^{2}+r^{2} \sin ^{2} \theta \dot{\phi}^{2}+\sigma c_{5}^{2} N^{2}\right]
$$

the equations of motion are

$$
\begin{aligned}
& 0=\ddot{t}+\frac{\partial_{r} B}{B} \dot{r} \dot{t}+c_{5} \frac{\partial_{5} B}{B} \dot{t}+\frac{1}{2} \sigma \frac{c_{5}^{2}}{c^{2}} \frac{\partial_{t} N^{2}}{B} \\
& 0=\ddot{r}+\frac{1}{2} \frac{\partial_{r} A}{A} \dot{r}^{2}+\frac{1}{2} c^{2} \frac{\partial_{r} B}{A} \dot{t}^{2}-\frac{1}{A} r \dot{\theta}^{2}-\frac{1}{A} r \sin ^{2} \theta \dot{\phi}^{2}+c_{5} \frac{\partial_{5} A}{A} \dot{r}-c_{5}^{2} \frac{1}{2} \sigma \frac{\partial_{r} N^{2}}{A} \\
& 0=r^{2} \ddot{\theta}+2 r \dot{r} \dot{\theta}-r^{2} \sin \theta \cos \theta \dot{\phi}^{2} \\
& 0=\ddot{\phi}+2 \frac{1}{r} \dot{r} \dot{\phi}+2 \cot \theta \dot{\theta} \dot{\phi}
\end{aligned}
$$

which are simplified using the rotational symmetry to put $\theta=\pi / 2$. Writing $\partial_{5}=\left(1 / c_{5}\right) \partial_{\tau}$, these become

$$
\begin{aligned}
& 0=\ddot{t}+\frac{\partial_{r} B}{B} \dot{r} \dot{t}+\frac{1}{2} \sigma \frac{c_{5}^{2}}{c^{2}} \frac{\partial_{t} N^{2}}{B} \\
& 0=\ddot{r}+\frac{1}{2} \frac{\partial_{r} A}{A} \dot{r}^{2}+\frac{1}{2} c^{2} \frac{\partial_{r} B}{A} \dot{t}^{2}-\frac{1}{A} r \dot{\phi}^{2}-c_{5}^{2} \frac{1}{2} \sigma \frac{\partial_{r} N^{2}}{A} \\
& 0=\ddot{\phi}+2 \frac{1}{r} \dot{r} \dot{\phi}
\end{aligned}
$$

where we used $\partial_{\tau} B=0$. The angular equation has the standard solution

$$
0=\ddot{\phi}+2 \frac{1}{r} \dot{r} \dot{\phi} \longrightarrow 0=\frac{\ddot{\phi}}{\dot{\phi}}+2 \frac{\dot{r}}{r} \longrightarrow r^{2} \dot{\phi}=J
$$

with constant $J$. In the equation for $t$ we recognize 


$$
\ddot{t}+\frac{\partial_{r} B}{B} \dot{r} \dot{t}=\frac{1}{B}\left(B \frac{d \dot{t}}{d \tau}+\frac{d B}{d \tau} \dot{t}\right)=\frac{1}{B} \frac{d(\dot{t} B)}{d \tau}
$$

and so (202) becomes

$$
0=\frac{d(\dot{t} B)}{d \tau}+\frac{1}{2} \sigma \frac{c_{5}^{2}}{c^{2}} \partial_{t} N^{2}
$$

leading to a perturbation in the evolution of the $t$ coordinate, which recovers the usual relation

$$
\dot{t}=\left(1-\frac{2 G m}{r c^{2}}\right)^{-1}
$$

for $\partial_{t} N^{2} \rightarrow 0$. It is convenient to rewrite (207) as

$$
\dot{t}=\frac{1}{B}\left(1-\sigma \frac{c_{5}^{2}}{c^{2}} \frac{1}{2} \int^{\tau} d \tau \partial_{t} N^{2}\right)
$$

Using (205) and (209), the radial equation becomes

$$
0=\ddot{r}+\frac{1}{2} \frac{\partial_{r} A}{A} \dot{r}^{2}+\frac{1}{2} c^{2} \frac{\partial_{r} B}{B}\left(1-\sigma \frac{c_{5}^{2}}{c^{2}} \frac{1}{2} \int^{\tau} d \tau \partial_{t} N^{2}\right)^{2}-\frac{1}{A} \frac{J^{2}}{r^{3}}-c_{5}^{2} \frac{1}{2} \sigma \frac{\partial_{r} N^{2}}{A}
$$

which we multiply by $2 A \dot{r}$ and use

$$
\begin{aligned}
\frac{d}{d \tau}\left(A \dot{r}^{2}\right) & =2 A \ddot{r} \ddot{r}+\dot{r}^{3} \partial_{r} A \\
\frac{d}{d \tau}\left(\frac{J^{2}}{r^{2}}\right) & =-2 \dot{r} \frac{J^{2}}{r^{3}} \\
\frac{d}{d \tau}\left(-\frac{1}{B}\right) & =\dot{r} \frac{\partial_{r} B}{B^{2}} \\
\frac{d}{d \tau} N^{2} & =\dot{r} \partial_{r} N^{2}+\dot{t} \partial_{t} N^{2}+\partial_{\tau} N^{2}
\end{aligned}
$$

to obtain

$$
0=\frac{d}{d \tau}\left[A \dot{r}^{2}-c^{2} \frac{1}{B}+\frac{J^{2}}{r^{2}}-c_{5}^{2} \sigma N^{2}\right]+\sigma c_{5}^{2}\left[\left(\frac{d}{d \tau} \frac{1}{B}\right) \int^{\tau} d \tau \partial_{t} N^{2}+\frac{1}{B} \partial_{t} N^{2}+\partial_{\tau} N^{2}\right]
$$

where we dropped terms in $c_{5}^{4}$. Integrating by parts

$$
\left(\frac{d}{d \tau} \frac{1}{B}\right) \int^{\tau} d \tau \partial_{t} N^{2}=\frac{d}{d \tau}\left(\frac{1}{B} \int^{\tau} d \tau \partial_{t} N^{2}\right)-\frac{1}{B} \partial_{t} N^{2}
$$

and so the radial Equation (215) becomes

$$
0=\frac{d}{d \tau}\left[A \dot{r}^{2}-c^{2} \frac{1}{B}+\frac{J^{2}}{r^{2}}-c_{5}^{2} \sigma\left(N^{2}-\frac{1}{B} \int^{\tau} d \tau \partial_{t} N^{2}\right)\right]+c_{5}^{2} \sigma \partial_{\tau} N^{2} .
$$

Using (37) the Hamiltonian in these coordinates is

$$
K=\frac{1}{2} M g_{\mu v} \dot{x}^{\mu} \dot{x}^{\nu}-\frac{1}{2} M c_{5}^{2} g_{55}=\frac{1}{2} M\left(A \dot{r}^{2}-c^{2} \frac{1}{B}+\frac{J^{2}}{r^{2}}\right)-\frac{1}{2} M c_{5}^{2} \sigma N^{2}
$$

and the radial equation is now 


$$
0=\frac{d}{d \tau}\left[\frac{K}{2 M}+c_{5}^{2} \sigma \frac{1}{B} \int^{\tau} d \tau \partial_{t} N^{2}\right]+c_{5}^{2} \sigma \partial_{\tau} N^{2}
$$

showing that the Hamiltonian, and thus the dynamical mass of the test particle, is not conserved. If, for example, we consider a very short perturbation, so that

$$
N(t, r, \tau)=\alpha(\tau) W(t, r)
$$

with $\alpha(\tau)$ a narrow distribution centered on $\tau=\tau_{0}$, then writing

$$
\Delta M=\sigma c_{5}^{2} \int d \tau \partial_{t} N^{2} \simeq \sigma c_{5}^{2} \partial_{t} W^{2}\left(t\left(\tau_{0}\right), r\left(\tau_{0}\right)\right) \int d \tau \alpha^{2}(\tau)
$$

we may integrate (219) to obtain

$$
\frac{K}{2 M}+\Delta M\left(1-\frac{2 G m}{r c^{2}}\right)^{-1} \simeq-c_{5}^{2} \sigma W^{2}\left(t\left(\tau_{0}\right), r\left(\tau_{0}\right)\right)\left[\alpha^{2}(\infty)-\alpha^{2}(-\infty)\right]=\kappa=\text { constant }
$$

describing a distance-dependent shift in the mass of the test particle. Thus, while the mass parameter $m$ that is associated with a source mass remains constant, the addition of a $g_{55}$ component to the metric induces mass transfer in the gravitational field.

\subsection{Variable Mass Source}

As a second example, we put $N=1$ and consider a $\tau$-dependent variation in the mass $M$ parameter of the metric, as given by

$$
M(\tau)=m[1+\alpha(\tau)]
$$

where the perturbation is small and so

$$
\alpha^{2} \ll 1 \longrightarrow B=A^{-1}=1-\Phi_{0}[1+\alpha(\tau)]
$$

where by comparison with (190) we have $\Phi_{0}=2 G m / r c^{2}$. The $4 \mathrm{D}$ connection is now $\tau$-dependent, but retains its unperturbed form with respect to the coordinates $x^{\mu}$, so the space remains Ricci flat with $\bar{R}=0$. We may ask what kind of mass-energy-momentum configuration would give rise to a Schwarzschild geometry with $\tau$-varying mass parameter, and if such a configuration can be made consistent with the Hamiltonian and momentum constraints. The dynamical equation for the metric (neglecting terms in $\alpha^{2}$ and $\Phi_{0}^{2}$ ) is

$$
\partial_{5} \gamma_{\mu \nu}=-2 N K_{\mu \nu} \longrightarrow K_{\mu \nu}=-\frac{1}{2 c_{5}} \partial_{\tau} \gamma_{\mu \nu}=-\frac{\Phi_{0} \dot{\alpha}(\tau)}{2 c_{5}} \operatorname{diag}\left(1, \frac{1}{B^{2}}, 0,0\right)
$$

and raising one index, we find

$$
K_{v}^{\mu}=\gamma^{\mu \lambda} K_{\lambda v}=-\frac{\Phi_{0} \dot{\alpha}(\tau)}{2 c_{5} B} \operatorname{diag}(-1,1,0,0) \longrightarrow K=K_{\mu}^{\mu}=0 .
$$

Using $\bar{R}=0, N=1, N^{\mu}=0,\left(K_{\mu v}\right)^{2} \propto \alpha^{2} \approx 0$, along with (225), the evolution equation for the extrinsic curvature can be written

$$
\frac{1}{c_{5}} \partial_{\tau} K_{\mu \nu}=-\frac{1}{2 c_{5}^{2}} \Phi_{0} \ddot{\alpha}(\tau) \operatorname{diag}\left(1, \frac{1}{B^{2}}, 0,0\right)=\sigma \frac{8 \pi G}{c^{4}}\left[S_{\mu \nu}-\frac{1}{2} \gamma_{\mu \nu}(S+\sigma \kappa)\right]
$$

which can be solved for $\alpha(\tau)$ if the energy-momentum tensor is known. Because $\bar{R}=K=0$ and $K^{\mu v} K_{\mu v} \propto \alpha^{2} \Phi_{0}^{2} \approx 0$, we may take the Hamiltonian constraint 


$$
\bar{R}-\sigma\left(K^{2}-K^{\mu v} K_{\mu v}\right)=-\sigma \frac{16 \pi G}{c^{4}} \kappa
$$

as the statement that the mass density $\kappa$ is approximately zero. Thus, the evolution Equation (227) for $K_{\mu v}$ can be satisfied by

$$
S_{00}=B^{2} S_{11}=\left(-\sigma \frac{c_{5}^{2}}{c^{2}} \frac{16 \pi G}{c^{2}}\right)^{-1} \Phi_{0} \ddot{\alpha}(\tau) \quad S_{22}=S_{33}=0 \longrightarrow S=0
$$

describing a $\tau$-dependent energy density $S_{00}$ and an energy-momentum $S_{11}$ flowing into the radial direction. Using the nonzero Christoffel symbols for the Schwarzschild metric

$$
\begin{array}{cc}
\Gamma_{10}^{0}=\frac{1}{2} \frac{\partial_{r} B}{B} & \Gamma_{00}^{1}=\frac{1}{2} \frac{\partial_{r} B}{A} \\
\Gamma_{11}^{1}=\frac{1}{2} \frac{\partial_{r} A}{A}=-\frac{1}{2} \frac{\partial_{r} B}{B} \quad \Gamma_{22}^{1}=-\frac{1}{A} r \quad \Gamma_{33}^{1}=-\frac{1}{A} r \\
\Gamma_{12}^{2}=\frac{1}{r} \quad \Gamma_{13}^{3}=\frac{1}{r}
\end{array}
$$

the momentum constraint

$$
p_{v}=D_{\mu} K_{v}^{\mu}-D_{v} K=\partial_{\mu} K_{v}^{\mu}+K_{v}^{\lambda} \Gamma_{\lambda \mu}^{\mu}-K_{\lambda}^{\mu} \Gamma_{v \mu}^{\lambda}
$$

has components

$$
\begin{aligned}
& p_{0}=\partial_{r} K_{0}^{1}+K_{0}^{0} \Gamma_{0 \mu}^{\mu}-K_{\lambda}^{\mu} \Gamma_{0 \mu}^{\lambda}=K_{0}^{0} \Gamma_{0 \mu}^{\mu}-K_{0}^{0} \Gamma_{00}^{0}-K_{1}^{1} \Gamma_{01}^{1}=0 \\
& p_{1}=\partial_{r} K_{1}^{1}+K_{1}^{\lambda} \Gamma_{\lambda \mu}^{\mu}-K_{\lambda}^{\mu} \Gamma_{1 \mu}^{\lambda}=-\frac{1}{2} \frac{1}{c_{5} r} \Phi_{0} \dot{\alpha}(\tau) \\
& p_{2}=p_{3}=0
\end{aligned}
$$

which corresponds to a mass current $p_{1}$ flowing in the radial direction, driving the varying mass parameter $M(\tau)$ in the metric. Ascribing $M(\tau)$ to a $\tau$-dependent mass distribution that produces the energy density $S_{00}$, energy-momentum $S_{11}$, and mass current $p_{1}$, we see once again that a variation in source mass will be transferred across spacetime by the induced gravitational field and, in turn, this field will lead to geodesic motion corresponding to varying mass in a test event.

\section{Discussion}

Stueckelberg-Horwitz-Piron (SHP) theory is a covariant approach to relativistic dynamics developed to address the problem of time as it arises in electrodynamics. In order to account for pair creation/annihilation processes in particular, and to remove from kinematics any a priori constraints that may lead to formal difficulties in describing relativistic interaction in general, SHP poses a theory of spacetime events $x^{\mu}$ occurring irreversibly at a chronological time $\tau$. By working through the implications of gauge theory at the classical and quantum levels, SHP introduces five $\tau$-dependent electromagnetic potentials that reduce to Maxwell fields at $\tau$-equilibrium. The equations of SHP electrodynamics suggest a formal 5D symmetry structure that must be broken to $4+1$ representations of $\mathrm{O}(3,1)$ on physical grounds. The resulting interactions form an integrable system in which event evolution generates an instantaneous current defined over spacetime at $\tau$, and, in turn, these currents induce $\tau$-dependent fields that act on other events at $\tau$.

In this paper, we extend these ideas into general relativity, posing a $5 \mathrm{D}$ pseudo-spacetime coordinatized by $\left(x^{\mu}, \tau\right)$ and possessing a formal 5D general diffeomorphism symmetry, which must similarly break to $4+1$ representations of geometrical and dynamical symmetries. This approach makes SHP general relativity naturally amenable to an unambiguous $4+1$ foliation, permitting a 
$\tau$-dependent generalization of gravitation that can be decomposed to a set of $4 \mathrm{D}$ curvature and matter distribution structures that evolve in $\tau$. We have shown that the 15 Einstein equations in $5 \mathrm{D}$ decompose into five constraints on initial conditions and 10 unconstrained evolution equations for the gravitational field, equivalent to removing the a priori constraints from the 10 Einstein equations in $4 \mathrm{D}$. It is the removal of these constraints that permit mass transfer in SHP gravitation, just as the absence of a mass-shell constraint permits the exchange of particles and fields in SHP electrodynamics. We completed the transformation of this system to an ADM-like canonical system, although computation is generally simpler in the system defined by the intrinsic and extrinsic curvatures. In analogy to SHP electrodynamics, the resulting formulation of general relativity describes an instantaneous distribution of mass and energy at $\tau$ expressed through $T_{\alpha \beta}(x, \tau)$, inducing a local metric $g_{\alpha \beta}(x, \tau)$, which, in turn, determines geodesic equations of motion for any particular event at $x^{\mu}(\tau)$.

As a simple first example of this method, we obtained a nonrelativistic generalization of Newtonian gravitation in the weak field approximation, by considering a $\tau$-dependent massive source. We saw that the non-constant source mass induces a $\tau$-dependent metric, that, in turn, leads to geodesic motion for a test event associated with non-conservation of the Hamiltonian function and, thus, mass variation. We then considered two generalizations of the Schwarzschild solution. In the first, we introduced a non-trivial metric component $g_{55}$ and saw that it must satisfy a $4 \mathrm{D}$ sourceless wave equation. This generalized plane wave similarly has the effect of inducing a mass shift in a test event. In a second generalization, we treated the mass parameter in the standard components of the Schwarzschild metric as $\tau$-dependent and solved for the matter distribution that would produce this perturbation. We found that the mass density of the matter distribution effectively vanishes, while the energy density and momentum density into the radial direction drive the variation of $M$. These mass effects may be compared to Equation (7), in which we saw that the SHP electromagnetic field component $f_{5 \alpha}$ permits the exchange of mass between particles and fields, and is, thus, the condition for non-conservation of proper time. The first term of

$$
f_{5 \alpha}=\partial_{5} a_{\alpha}-\partial_{\alpha} a_{5}
$$

induces mass exchange through $\tau$-dependence of the electromagnetic field $a_{\alpha}$, in analogy with the $\tau$-dependent gravitational field $\gamma_{\mu \nu}(x, \tau)$ seen in (59) and (190). The second term induces mass exchange through a non-trivial fifth field component $a_{5}$, in analogy with the $g_{55}$ metric component used in (196).

Beyond the theoretical interest in modified gravity, the $4+1$ formalism in SHP general relativity offers a potentially significant tool for the calculation of complex dynamics in numerical relativity. For example, the weak field approximation for a single source event given in Section 2.5 may be extended by introducing a second source moving at nonrelativistic velocity toward the first. Although the equations of motion for a test particle in the resulting field is not amenable to closed form solution, a straightforward numerical solution will take account of the nonlinear evolution of the particle's coordinate time $t$. By comparison, a solution using $x^{0}=c t$ as the evolution parameter for the particles and fields will necessarily involve significantly more computational complexity.

It has been shown [31] that Maxwell theory emerges from SHP electrodynamics by taking $c_{5} \rightarrow 0$ and, in this sense, can be seen as an equilibrium limit as $\tau$-evolution of the field reaches a steady state. Alternatively, this limit is obtained, under appropriate boundary conditions, by integration [5] of the fields and currents over $\tau$, with the effect of summing at each spacetime point the contributions from all values of $\tau$. At equilibrium, the electromagnetic fields become $\tau$-independent and the fields associated with the potential $a_{5}$ decouple from matter, so that, while proper time remains unconstrained, it behaves as a classical conserved quantity. Thus, by restricting his electromagnetic formalism to the $\tau$-independent four-vector potential $A^{\mu}(x)$, Fock remained within standard Maxwell theory. This restriction was also applied in the formulations of quantum electrodynamics (QED) by Schwinger [32] and Feynman [33], leading to fixed masses for asymptotic particle states. 
As we saw in Equation (155) the Einstein tensor in SHP can be split into ten unconstrained components and five constraints among initial condition. It remains to be shown that the unconstrained components correspond to the ten components of the Einstein tensor in $4 \mathrm{D}$, and the five constraints permit mass exchange but conserve the total mass of matter and fields. We further expect that, as in electrodynamics, an appropriate restriction in SHP GR will lead to a decoupling of field components and the conservation of four of the ten components of the Einstein tensor. This restriction should produce a $\tau$-parameterized formulation of standard 4D GR, analogous to Fock's proper-time formulation of Maxwell theory. While it is evident from (34) that taking $c_{5}$ to zero recovers the standard spacetime geodesic Equation (35), the nonlinearity of the Einstein field equations makes the problem of extracting a $\tau$-parameterized field theory considerably more difficult. These aspects of the theory will be reported in future work. Such a theory would be especially useful in cases of strong gravitational fields. We thus expect that calculations of black hole collisions and radiation from stellar collapse may be improved by posing the initial value problem with respect to the external evolution parameter $\tau$. Numerical calculations of this type are beyond the scope of this paper and they will be discussed in future work.

Funding: This research received no external funding.

Acknowledgments: It is my pleasure to dedicate this paper to Lawrence P. Horwitz-teacher, collaborator, and inspiration —on the occasion of his 90th birthday.

Conflicts of Interest: The author declares no conflict of interest.

\section{References}

1. Arnowitt, R.L.; Deser, S.; Misner, C.W. Republication of: The dynamics of general relativity. Gen. Relativ. Gravit. 2004, 40, 1997-2027. [CrossRef]

2. Stueckelberg, E. La signification du temps propre en mécanique: Ondulatoire. Helv. Phys. Acta 1941, 14, 321-322. (In French)

3. Stueckelberg, E. Remarque a propos de la création de paires de particules en théorie de relativité. Helv. Phys. Acta 1941, 14, 588-594. (In French)

4. Horwitz, L.; Piron, C. Relativistic Dynamics. Helv. Phys. Acta 1973, 48, 316-326.

5. Saad, D.; Horwitz, L.; Arshansky, R. Off-shell electromagnetism in manifestly covariant relativistic quantum mechanics. Found. Phys. 1989, 19, 1125-1149. [CrossRef]

6. Horwitz, L.P. Relativistic Quantum Mechanics; Springer: Dordrecht, The Netherlands, 2015. [CrossRef]

7. Land, M.; Horwitz, L.P. Relativistic Classical Mechanics and Electrodynamics; Morgan and Claypool Publishers: San Rafae, CA, USA, 2020. [CrossRef]

8. Horwitz, L.P. An Elementary Canonical Classical and Quantum Dynamics for General Relativity. J. Phys. Conf. Ser. 2019, 1239, 012014. [CrossRef]

9. Horwitz, L.P. An elementary canonical classical and quantum dynamics for general relativity. Eur. Phys. J. Plus 2019, 134, 313. [CrossRef]

10. Wheeler, J.A. Geons, Black Holes and Quantum Foam: A Life in Physics; W. W. Norton \& Company: New York, NY, USA, 2000.

11. Isham, C. Canonical Quantum Gravity and the Problem of Time; Technical Report Imperial/TP/91-92/25; Lectures at the NATO Summer School in Salamanca; Blackett Laboratory, Imperial College: London, UK, 1992.

12. Kiefer, C. Space, Time, Matter in Quantum Gravity. In Hundred Years of Gauge Theory; De Bianchi, S., Kiefer, C., Eds.; Springer: New York, NY, USA, 2020.

13. Wheeler, J.A. Superspace and the nature of quantum geometrodynamics. In Topics in Nonlinear Physics; Zabusky Norman, J., Ed.; Springer: New York, NY, USA, 1969; pp. 615-724.

14. Fock, V. Proper time in classical and quantum mechanics. Phys. Z. Sowjetunion 1937, 12, 404-425.

15. Land, M.; Shnerb, N.; Horwitz, L. On Feynman's approach to the foundations of gauge theory. J. Math. Phys. 1995, 36, 3263. [CrossRef] 
16. Land, M.; Horwitz, L. The Lorentz Force and Energy-Momentum for Off-Shell Electromagnetism. Found. Phys. Lett. 1991, 4, 61. [CrossRef]

17. Land, M. Pair production in classical Stueckelberg-Horwitz-Piron electrodynamics. J. Phys. Conf. Ser. 2015, 615, 012007. [CrossRef]

18. Pitts, J.B.; Schieve, W.C. On Parametrized General Relativity. Found. Phys. 1998, 28, 1417-1424. [CrossRef]

19. Pitts, J.B.; Schieve, W.C. Flat Spacetime Gravitation with a Preferred Foliation. Found. Phys. 2001, 31, 1083-1104. [CrossRef]

20. Dirac, P. General Theory of Relativity; Princeton University Press: Princeton, NJ, USA, 1996.

21. Weinberg, S. Gravitation and Cosmology: Principles and Applications of the General Theory of Relativity; Wiley: New York, NY, USA, 1972.

22. Wald, R.M. General Relativity; Chicago Univ. Press: Chicago, IL, USA, 1984.

23. Land, M. Mass-Energy-Momentum Radiation in Stueckelberg-Horwitz-Piron (SHP) Electrodynamics. J. Phys. Conf. Ser. 2019, 1239, 012005. [CrossRef]

24. Misner, C.W.; Thorne, K.S.; Wheeler, J.A. Gravitation; W.H. Freeman and Co.: San Francisco, CA, USA, 1973.

25. Land, M. Local metric with parameterized evolution. Astron. Nachrichten 2019, 340, 983-988. [CrossRef]

26. Land, M.; Horwitz, L. Green's functions for off-shell electromagnetism and spacelike correlations. Found. Phys. 1991, 21, 299-310. [CrossRef]

27. Bertschinger, E. Hamiltonian Formulation of General Relativity; Technical Report Physics 8.962; Massachusetts Institute of Technology: Cambridge, MA, USA, 2002.

28. Zilhão, M. New frontiers in Numerical Relativity. arXiv 2013, arXiv:gr-qc/1301.1509.

29. Gourgoulhon, E. 3+1 Formalism and Bases of Numerical Relativity. arXiv 2007, arXiv:gr-qc/0703035.

30. Blau, M. Lecture Notes on General Relativity; Technical Report; Albert Einstein Center for Fundamental Physics, Universität Bern: Bern, Switzerland, 2020.

31. Land, M. Speeds of light in Stueckelberg-Horwitz-Piron electrodynamics. J. Phys. Conf. Ser. 2017, 845, 012024. [CrossRef]

32. Schwinger, J. On Gauge Invariance and Vacuum Polarization. Phys. Rev. 1951, 82, 664-679. [CrossRef]

33. Feynman, R. Mathematical formulation of the quantum theory of electromagnetic interaction. Phys. Rev. 1950, 80, 440-457. [CrossRef]

Publisher's Note: MDPI stays neutral with regard to jurisdictional claims in published maps and institutional affiliations.

(C) 2020 by the author. Licensee MDPI, Basel, Switzerland. This article is an open access article distributed under the terms and conditions of the Creative Commons Attribution (CC BY) license (http://creativecommons.org/licenses/by/4.0/). 